\title{
Membrane vesicles from the probiotic Nissle 1917 and gut resident Escherichia coli strains distinctly modulate human dendritic cells and subsequent $T$ cell responses
}

\author{
Natalia Diaz-Garrido ${ }^{1,2}$, María-José Fábrega ${ }^{1,2 \ddagger}$, Rodrigo Vera ${ }^{1,2}$, Rosa Giménez ${ }^{1,2}$, \\ Josefa Badia $^{1,2^{*}}$ and Laura Baldomà ${ }^{1,2^{*}}$ \\ ${ }^{1}$ Secció de Bioquímica i Biología Molecular, Departament de Bioquímica i Fisiologia, \\ Facultat de Farmàcia, Universitat de Barcelona, Barcelona, Spain. \\ ${ }^{2}$ Institut de Biomedicina de la Universitat de Barcelona, Institut de Recerca Sant Joan \\ De Déu (IR-SJD), Barcelona, Spain. \\ † Present address: Department of Experimental and Health Sciences. Parc de Recerca \\ Biomèdica de Barcelona. University Pompeu Fabra (UPF). Barcelona, Spain \\ * Both authors contributed equally to the supervision of the study.
}

Correspondence:

Laura Baldomà: lbaldoma@ub.edu 


\section{Abstract}

2 Extracellular membrane vesicles (MVs) released by gut microbiota are key players in 3 the communication with the host. The aim of this study was to evaluate the 4 immunomodulatory properties of MVs from the probiotic E. coli Nissle $1917(\mathrm{EcN})$ in 5 terms of DC-derived adaptative immune responses and to compare the effects with 6 those elicited by commensal E. coli. The effects of MVs were analysed in monocyte7 derived DCs by measuring cytokine expression and the ability of activated-DCs to 8 differentiate CD4+ T cells towards specific effector subsets. EcN MVs drived intricated 9 Th1/Th2/Th17/Th22/Treg responses consistent with the beneficial effects of this 10 probiotic. Th2/Th17/Th22 responses were common to commensal E. coli-derived vesicles but specific differences were observed for Th1 and Treg responses. Since MVs activate DCs in a strain-specific manner, probiotic-derived MVs could be explored as a safe (bacteria-free) strategy to develop new functional food ingredients targeting gut microbiota balance or intestinal inflammation.

Key words: Escherichia coli, Nissle 1917, gut microbiota, dendritic cells, membrane vesicles, innate immunity

Abbreviations: DCs: dendritic cells, DMEM: Dulbecco's modified Eagle medium, EcN: Escherichia coli Nissle 1917, FBS: foetal bovine serum, HPRT-1 hypoxanthine phosphoribosyl transferase, iDCs: immature DCs, LB: Luria-Bertani broth, LPS: lipopolysaccharide, MAMPs: microbial-associated molecular patterns, mDCs, mature DCs, Mo-DCs: monocyte-derived DCs, MFI: mean fluorescence intensity, MTT: 3(4,5-Dimethylthiazol-2-yl)-2,5-diphenyl tetrazolium bromide, MVs: membrane vesicles, NLR: NOD-like receptor, NOD: nucleotide oligomerization domain, OMVs: outer membrane vesicles, PBS: phosphate buffer saline; PRR: pattern recognition receptor, RT-qPCR: quantitative reverse transcription PCR, TEM: transmission electron microscopy, Th: T helper, TLR- Toll-like receptor, ZO: zonula occludens. 


\section{Introduction}

The human gastrointestinal tract is colonized by a diverse and complex microbial community known as gut microbiota, which is essential to intestinal homeostasis and human health. Besides its contribution to food digestion and nutrient metabolism, gut microbiota plays a pivotal role in host immune system development and in the modulation of gut barrier, immune and defence responses (Jandhyala et al., 2015; Thursby \& Juge, 2017). Variations in microbiota composition and diversity (dysbiosis) may disturb host balanced responses and contribute to a wide variety of diseases including cancer and inflammatory, autoimmune, metabolic and neurological disorders (Belkaid \& Hand, 2014; Gopalakrishnan et al., 2018; Baothman et al., 2016; Marin et al., 2017). The high plasticity of the human microbiome provides the basis for new therapeutic strategies aimed at restoring altered gut microbiota balance (Jia et al., 2008; Shanahan, 2011; Maguire \& Maguire, 2018). Administration of probiotics is one of these therapeutic interventions, which essentially attempts to take advantage of the beneficial effects of commensal microbiota.

Gut microbiota establishes dynamic, reciprocal interactions with the intestinal epithelium and the immune system. This complex network orchestrates appropriate immune responses that protect against enteric pathogens while maintaining tolerance to symbiotic microbiota and innocuous antigens. Constant stimulation of the intestinal immune system by commensal microbiota tightly regulates innate and adaptive responses, leading to controlled inflammatory responses that contribute to the training of immune system. Immune priming by microbiota is an essential mechanism to trigger appropriate immune responses against invading microbes to assist pathogen eradication and host survival (Belkaid \& Hand, 2014; Caballero \& Pamer, 2015).

The ability of intestinal cells to discriminate between pathogens and commensals is key to intestinal homeostasis. Dendritic cells (DCs) in the lamina propria play a pivotal role in sampling gut microbes and shaping appropriate immune responses. In fact, DCs serve as the cellular link between innate and adaptive responses. DCs are antigen-presenting cells that can contact the luminal environment through the inner mucosal lining. Recognition of gut microbes by these phagocytic cells depends on the expression of pattern-recognition receptors (PRRs) that interact with specific microbial components, known as microbial-associated molecular patterns (MAMPs). Upon ligand recognition, surface-located PRRs elicit intracellular signalling events that lead to 
bacterial phagocytosis and the expression of specific surface structures, co-stimulatory molecules and cytokines. Through antigen presentation and secretion of immune mediators, mature DCs (mDCs) act as messengers for the rest of the immune system, promoting differentiation of $\mathrm{T}$ helper $(\mathrm{Th})$ cells to specific $\mathrm{T} \mathrm{CD} 4^{+}$functional subsets (Hooper et al., 2012). Microbial activation of DCs towards secretion of TGF- $\beta$ and IL10 promotes generation of anti-inflammatory Foxp $3^{+}$regulatory $\mathrm{T}$ cells (Treg), which are involved in immunological tolerance to gut microbiota. In contrast, secretion of IL-6 and IL-1 $\beta$ by activated DCs predominantly induces proliferation of IL-17-secreting Th17 cells, which are crucial for the host defence response to bacterial pathogens and fungi. Th1 cells also have pro-inflammatory and protective functions against virus and bacterial pathogens. This cell subset releases INF- $\gamma$ and TNF- $\alpha$ and its expansion is mainly driven by DC-secreted IL-12. Secretion of IL-4 by activated DCs results in Th differentiation towards the Th2 subpopulation that secretes IL-4, IL-5, IL-13 and the anti-inflammatory IL-10. The Th2 response is predominantly activated against intestinal nematode parasites but an excessive, dysregulated Th2 propagation can promote allergic responses. Intestinal homeostasis and human health depend on well balanced Th17/Treg and Th1/Th2 responses. In this context, DCs ensure intestinal homeostasis by tuning the host immune system in response to gut microbes (Maynard et al., 2012).

In addition to DC interaction with bacteria that reach the gut-associated lymphoid tissue via transcytosis across M cells, DCs also sample gut luminal content by extending pseudopodia across the epithelial cell layer (Hooper et al., 2012). Under healthy conditions, the intestinal epithelium is covered by a mucus layer that maintains segregation of microbiota and host cells. Commensal microbiota inhabits the outer mucus layer, whereas the inner mucin layer is highly compacted and prevents bacteria from gaining access to the epithelial cells (Johansson et al., 2008; Vaishnava et al., 2011). Due to the gut barrier structure, microbiota-host communication is mainly mediated by secreted bacterial factors that can diffuse through the mucus layer and interact with cells of the intestinal mucosa. Besides metabolites and soluble secreted proteins, bacteria also release active mediators through extracellular membrane vesicles (MVs).

There is growing body of evidence that microbiota-derived MVs are key players in inter-kingdom communication as mediators of the effects of gut resident bacteria on host immune and defence responses (Kaparakis-Liaskos \& Ferrero, 2015). In this context, our group was a pioneer in the study of MVs from Escherichia coli. This 
bacterial species is a normal constituent of the gut microbiota. Certain intestinal E. coli isolates such as the strain Nissle 1917 (EcN) (Nissle, 1916; Sonnenborn \& Schulze, 2009) have probiotic properties while others are considered commensal strains although they have pathogen-like genes. The human gut is a reservoir of these pathobionts, which mainly fit in the E. coli phylogenetic group B2 that comprises strains that cause extraintestinal infections. Although it is a safe probiotic, EcN belongs to the phylogenetic group B2 (STEC Center, http://www.shigatox.net/new/reference-strains/ ecor.html) and shares a common ancestor with uropathogenic E. coli strains. During evolution, EcN lost virulence factors but conserved genes encoding fitness factors that contribute to its competitiveness and survival in the densely populated human gut (Vejborg et al., 2010; Toloza et al., 2015). This probiotic interferes with enteric pathogens and displays antiinflammatory and barrier strengthening activities. The immunomodulatory properties of $\mathrm{EcN}$ have been associated with the synthesis of colibactin (Olier et al., 2012) and the expression of a K5 capsule, which in addition mediates interactions with intestinal epithelial cells (Hafez et al., 2009; Hafez et al., 2010, Hafez, 2012).

The effects of microbiota MVs depend on their bacterial origin and cargo. Consequently, some of them are strain specific. For instance, MVs released by the gut symbiont Bacteroides fragilis induce Treg cells and mucosal tolerance through the delivery of the immunomodulatory polysaccharide A (Shen et al., 2012). Our studies showed that MVs isolated from the probiotic EcN (group B2) and the commensal ECOR12 (group A) and ECOR63 (group B2) are internalized by intestinal epithelial cells via clathrin-mediated endocytosis (Cañas et al., 2016) and modulate host immune and defence responses. In intestinal epithelial cells, internalized MVs activate NOD1dependent signalling pathways and contribute to microbiota-driven basal inflammation (Cañas et al., 2018). In addition, MVs from EcN and ECOR12 exhibit immunomodulatory effects on human colonic explants and in cellular models that mimic the intestinal epithelial barrier. In fact, these vesicles activate the synthesis of antimicrobial peptides and modulate the expression of inflammatory mediators towards an anti-inflammatory profile (Fábrega et al., 2016). Concerning barrier protection effects, MVs from EcN and ECOR63 reinforce the intestinal epithelial barrier and reduce gut permeability by modulating the tight junction proteins $\mathrm{ZO}-1$, claudin-14 and claudin-2 (Alvarez et al., 2016). The anti-inflammatory and barrier strengthening activities of EcN MVs were proven in in vivo models of experimental colitis. Oral 
administration of MVs isolated from this probiotic ameliorate inflammation and colitis progression in dextran sodium sulphate-treated mice (Fábrega et al., 2017).

The aim of this study was to evaluate the ability of MVs released by probiotic and commensal E. coli strains that belong to different phylogenetic groups to modulate DC function in vitro. We selected from the E. coli reference collection ECOR strain ECOR12 (group A) and strains ECOR63 and ECOR53 (group B2). Unlike ECOR63, strain ECOR53 expresses functional haemolysin and causes cytotoxicity in the murine macrophage cell line J774 (Lai et al., 1999). MVs isolated from an EcN K5-deficient mutant were also analysed to test whether the $\mathrm{K} 5$ capsular polysaccharide mediates $\mathrm{EcN}$ immunomodulatory effects in DCs. The vesicle-modulating properties were analysed in monocyte-derived DCs (mo-DCs) by measuring the cytokine release profile and the ability of induced-DCs to differentially modulate activation of $\mathrm{CD} 4^{+} \mathrm{T}$ cells towards Treg, Th17 and Th1 effector responses.

\section{Materials and methods}

\subsection{Bacterial Strains and Growth Conditions}

The probiotic strain EcN (serotype O6:K5:H1) was provided by Ardeypharm (GmbH, Herdecke, Germany). The EcN knockout mutant lacking the K5 capsule (EcN:K5) was kindly provided by I. Roberts. This mutant was generated by replacement of the $\mathrm{kfiC}$ gene with a chloramphenicol resistance cassette (Hafez et al., 2009). ECOR12, ECOR63 and ECOR53 are E. coli strains from the ECOR reference collection that were isolated from stool samples of healthy human donors (Ochman \& Selander, 1984). Bacterial strains were grown in Luria-Bertani broth (LB) at $37^{\circ} \mathrm{C}$, with constant rotation $(150 \mathrm{rpm})$. The EcN:K5 mutant strain was routinely grown on LBchloramphenicol, except for MV isolation. In that case, the culture medium was antibiotic-free LB to maintain equal growth conditions for all strains. Growth was monitored by measuring the optical density at $600 \mathrm{~nm}$.

\subsection{Isolation of MVs and Cryo-TEM analysis}

MVs were isolated from culture supernatants as described previously (Aguilera et al., 2014). Briefly, E. coli strains were grown overnight in LB and the culture was centrifuged $\left(10,000 \times \mathrm{g}, 20 \mathrm{~min}, 4^{\circ} \mathrm{C}\right)$ to remove bacteria. Culture supernatants were 
162 then filtered through a $0.22 \mu \mathrm{m}$-pore-size filter (Merck Millipore, Massachusetts, USA) and concentrated by centrifugation in a Centricon Plus-70 filter device (Merck Millipore, Massachusetts, USA). After an additional filtration step, vesicles were collected by centrifugation at $150,000 \times \mathrm{g}$ for $1 \mathrm{~h}$ at $4^{\circ} \mathrm{C}$, washed and resuspended in PBS. Quantification of MV samples was assessed by protein concentration and sterility was confirmed on LB plates. Aliquots of MVs were stored at $-20^{\circ} \mathrm{C}$ until use.

Cryo-transmission electron microscopy (Cryo-TEM) analysis was performed with fresh MVs samples resuspended in $0.1 \mathrm{M}$ phosphate buffer $(\mathrm{pH}$ 7.2) as described previously (Perez-Cruz et al., 2016). Briefly, one drop of each MV suspension (5 $\mu$ l) was applied on the carbon surface of a glow-discharged Lacey Carbon 300 mesh copper grid (Ted Pella, USA). The sample was allowing to adsorb for 4 min at $100 \%$ humidity inside the chamber of the Vitrobot Mark III (FEI Company, Eindhoven, Netherlands). The excess of liquid was automatically blotted with filter paper, followed by cryoimmobilization by plunge freezing in liquefied ethane. The vitrified sample was stored in liquid nitrogen until its observation in the cryo-electron microscope. Plunge-frozen sample was transferred to a Tecnai F20 EM (FEI, Eindhoven, The Netherlands) using a cryo-holder system (Gatan, Pleasanton, USA). The sample was examined at $200 \mathrm{kV}$, at a temperature ranging from -179 to $-170^{\circ} \mathrm{C}$, using low-dose imaging conditions. Lowdose images were recorded with a $4096 \times 4096$-pixel CCD Eagle camera (FEI, Eindhoven, The Netherlands).

\subsection{Generation of human monocyte-derived DCs}

Human mo-DCs were generated in vitro as described elsewhere (Lopez et al., 2012). Briefly, peripheral blood mononuclear cells (PBMCs) were isolated from fresh buffy coats of healthy donors, provided by the "Banc de Sang i Teixits" of Barcelona according to the signed agreement with the Institution. Use of anonymous, nonidentifiable human samples was approved by the Bioethics Committee of the University of Barcelona (Institutional Review Board:1R800003099). Human PBMCs were isolated by density gradient centrifugation using Histopaque 1077 (Sigma-Aldrich Chemical Co, St. Louis, USA) following the manufacturer's instructions. Monocytes were isolated from PBMCs by positive selection using magnetic-activated cell sorting with CD14 microbeads (Miltenyi Biotec, Bergish Gladbach, Germany) and cultured at density 2 x $10^{5}$ cells $/ \mathrm{ml}$ in 12 -well plates for 6 days $\left(37^{\circ} \mathrm{C}, 5 \% \mathrm{CO}_{2}\right)$ in complete MO-DC 
195 differentiation medium that contains $800 \mathrm{IU} / \mathrm{ml}$ GM-CSF and $1000 \mathrm{IU} / \mathrm{ml}$ IL-4 196 (Miltenyi Biotec, Bergish Gladbach, Germany). Fresh complete MO-DC medium

197

198

199

200

201

202

203

204

205

206

207

208

209

210

211

212

213

214

215

216

217

218

219

220

221

222

223

224

225

226

227 containing GM-CSF and IL-4 was added the fourth day of culture.

\subsection{Mo-DCs stimulation and maturation}

On day 6, immature mo-DCs (iDCs) were washed and kept in fresh MO-DC differentiation medium for at least one hour at $37^{\circ} \mathrm{C}$ in $5 \% \mathrm{CO}_{2}$ before the addition of MVs $(10 \mu \mathrm{g} / \mathrm{ml})$. After 24-hour incubation with $\mathrm{MVs}$ at $37^{\circ} \mathrm{C}$ in $5 \% \mathrm{CO}_{2}$, maturation of dendritic cells was verified by flow cytometry analysis of surface maturation markers. DC supernatants were sterile filtered through a $0.2 \mu \mathrm{m}$ pore filter and stored at $-80^{\circ} \mathrm{C}$ for cytokine quantification.

\subsection{Isolation of $C D 4^{+}$T cells from PBMCs and DCs $/ C D 4^{+} T$ cells co-culture}

$\mathrm{CD}^{+} \mathrm{T}$ cells were isolated from human PBMCs by positive selection using MACS CD4 ${ }^{+} \mathrm{T}$ Cell Isolation Kit (Miltenyi Biotec, Bergish Gladbach, Germany) and resuspended in fresh complete TexMACs ${ }^{\mathrm{TM}}$ media (Miltenyi Biotec, Bergish Gladbach, Germany) at a density of $1 \times 10^{6}$ cells $/ \mathrm{ml}$. CD4 ${ }^{+} \mathrm{T}$ cell purity was greater than $95 \%$. Then, DCs stimulated for $24 \mathrm{~h}$ with MVs (mature DCs, mDCs) were washed and cocultured with isolated allogenic $\mathrm{CD}^{+} \mathrm{T}$ cells in 12-well plates at a DC:T cell ratio of 1:5 ( $2 \times 10^{5} \mathrm{DCs} / 1 \times 10^{6} \mathrm{~T}$ cells) in TexMACs medium (Höpken et al. 2005). Single cultures of $\mathrm{CD}^{+} \mathrm{T}$ cells and $\mathrm{iDC} / \mathrm{CD} 4^{+} \mathrm{T}$ cell co-cultures were processed in parallel as a control. After 3 days incubation, culture supernatants were collected, centrifuged and filtered for cytokine quantification, and pelleted lymphocytes were stained for flow cytometry analysis of Th17 $\left(\mathrm{CD} 196^{+}\right)$and Treg $\left(\mathrm{CD} 25\right.$ (high), FoxP3 $\left.{ }^{+}\right)$phenotypes.

\subsection{Stimulation of mo-DCs across Caco-2 cell monolayers}

The intestinal epithelial cell line Caco-2 (ATCC HTB37) was routinely cultured in DMEM High Glucose supplemented with $10 \%$ foetal bovine serum (FBS), $25 \mathrm{mM}$ HEPES, 1\% non-essential amino acids and penicillin/streptomycin. For experiments, Caco-2 cells $\left(1 \times 10^{5}\right.$ cells $\left./ \mathrm{cm}^{2}\right)$ were seeded on the apical surface of polycarbonate Transwell filter inserts (12 mm, 0.4- $\mu \mathrm{m}$-pores; Millipore, Massachusetts, USA) and cultured for 15-18 days with medium changes on alternate days. Monolayer integrity was assessed by transepithelial electrical resistance (TER) and microscopic evaluation 
as previously reported (Alvarez et al. 2016). Activation of Caco-2 monolayers with 229 MVs was performed at TER values greater than $800 \Omega . c m 2$. After PBS washing, Caco2 monolayers were kept in fresh medium, and apically stimulated with MVs $(10 \mu \mathrm{g} / \mathrm{ml})$ for $24 \mathrm{~h}$. Some wells were left untreated as a control. Culture supernatants were then collected from the basolateral compartments and used to activate mo-DCs $\left(1 \times 10^{6}\right.$ cells/ml). After $24 \mathrm{~h}$ incubation, the DC culture supernatant was collected for quantification of secreted cytokines.

2.7. Flow Cytometry

Maturation of vesicle-stimulated mo-DCs was verified by flow cytometry using the MO-DC Differentiation Inspector Kit (Miltenyi Biotec, Bergish Gladbach, Germany). Briefly, cells were centrifuged (200 x g, $5 \mathrm{~min}$ ) and stained for $15 \mathrm{~min}$ at $4^{\circ} \mathrm{C}$ with monoclonal antibody (mAb) anti-CD14 fluorescein isothiocyanate (FITC) (clone Tuk4), anti-CD209 R-phycoerythrin (PE) (clone DCN-47.5.4) and anti-CD83 allophycocyanin (APC) (clone HB15) following the manufacturer's protocol. A human control cocktail of isotype matched conjugated irrelevant mAbs was used as a negative control. Cells were resuspended in PBS 2\% BSA and kept on ice until flow cytometric analysis. Stained cells (at least 10,000 events) were acquired on a Gallios flow cytometer (Beckman Coulter, Inc, Fullerton, CA, USA) with a 3-laser-10 colour standard configuration.

After 3 days co-incubation of vesicle-stimulated mo-DCs with $\mathrm{CD}^{+}{ }^{+} \mathrm{T}$ cells, lymphocytes were collected, washed and resuspended in PBS 2\% BSA for Th17 and Treg phenotypic analysis. For surface marker staining, lymphocytes were first stained with anti-CD4 FITC (clone VIT4), anti-CD25 APC (clone 4E3) and anti-CD196 PE (clone REA190) mAb or with the corresponding isotype-matched conjugated irrelevant antibody (Miltenyi Biotec, Bergish Gladbach, Germany). Then, cells were fixed, permeabilized and intracellularly stained with anti-FoxP3 PE (clone 3G3) (Miltenyi Biotec, Bergish Gladbach, Germany) or with the corresponding isotype-matched control, following the manufacturer's instructions. A minimum of 10,000 stained CD4 ${ }^{+}$ lymphocytes were acquired on a Gallios flow cytometer. The specific fluorescence intensity was quantified as the mean fluorescence intensity (MFI) calculated by subtracting the background of isotype matched control staining from the total fluorescence. 


\subsection{Cell Viability assays}

Cell viability was assessed by flow cytometry using the exclusion dye 4',6266 diamidino-2-phenylindole (DAPI) violet. In addition, the trypan blue exclusion test and the MTT (3-(4,5-Dimethylthiazol-2-yl)-2,5-diphenyl tetrazolium bromide) assay were used to evaluate cell viability in vesicle-stimulated DCs. For this, $1 \times 10^{5}$ mo-DCs in 100 $\mu \mathrm{l}$ of media were plated into each well in a 96-well plate and incubated for $24 \mathrm{~h}$ at $37^{\circ} \mathrm{C}$ before the addition of MVs $(10 \mu \mathrm{g} / \mathrm{ml})$. After $24 \mathrm{~h}$-incubation, cells were treated with $0.25 \%$ MTT (Sigma-Aldrich Chemical Co, St. Louis, MO, USA) in PBS and allowed to react for $2 \mathrm{~h}$ at $37^{\circ} \mathrm{C}$. The medium was then removed and $0.1 \mathrm{ml}$ of solubilization reagent (99\% dimethyl sulfoxide) was added. Cell viability was measured at $570 \mathrm{~nm}$ in a Modulus $^{\mathrm{TM}}$ Microplate Photometer (Turner BioSystems Inc., Sunnyvale, CA, USA).

\subsection{RNA isolation and quantitative reverse transcription-PCR $(R T-q P C R)$}

Total RNA was extracted from mo-DCs using a Qiagen RNeasy Mini Kit 280 according to the manufacturer's instructions (Qiagen, Crawley, UK). RNA quality and concentration were assessed by the ratio of absorbance at 260 and $280 \mathrm{~nm}$ using a NanoDrop TM-2000 spectrophotometer (Thermo Fisher Scientific, Waltham, Massachusetts, USA). RNA integrity was verified by visualization of $28 \mathrm{~S}$ and $18 \mathrm{~S}$ rRNAs after $1 \%$ agarose/formaldehyde gel electrophoresis.

For RT-qPCR, $1 \mu \mathrm{g}$ RNA was reverse transcribed using the High Capacity cDNA Reverse Transcription kit (Applied Biosystems, Foster City, CA, USA) in a final volume of $20 \mu \mathrm{l}$. Quantitative PCR reactions were performed in a StepOne Plus PCR cycler (Applied Biosystems, Foster City, CA, USA) by using SYBR® Green PCR Master Mix (Applied Biosystems, Foster City, CA, USA) and specific oligonucleotides for IL-6, TNF- $\alpha$, IL-10 and TGF- $\beta$ (Table 1). The housekeeping HPRT-1 gene was used as a normalizing gene. A control reaction was performed in the absence of RNA. The standard PCR program was: one denaturation cycle for $10 \mathrm{~min}$ at $95^{\circ} \mathrm{C}$ followed by 40 cycles of $15 \mathrm{~s}$ at $95^{\circ} \mathrm{C}$ and $1 \mathrm{~min}$ at $60^{\circ} \mathrm{C}$. Relative gene expression was calculated as 
294 fold change compared with control and calculated by means of $2^{-\Delta \Delta C t}$ formula (Livak \& 295 Schmittgen, 2001).

296

297

\subsection{Cytokine quantification}

Secreted cytokines, IFN $\gamma$, IL-1 $\beta$, TNF- $\alpha$, IL-12p70, IL-17A, IL-18, IL-22, IL-23, IL-4, IL-5, IL-13, IL-10, and TGF- $\beta$ were quantified in culture supernatants by the 300 human ProcartaPlex Multiplex Immunoassay (eBiosciences Inc., San Diego, CA, USA) according to manufacturer's instructions. Concentration of each analyte was detected using the MAGPIX instrument (Luminex Corp., Austin, TX, USA) in the facilities of the Scientific and Technological Centres of the University of Barcelona (CCiT-UB), and results were analysed with xPONENT ${ }^{\circledR} 4.2$ software (Luminex Corp., Austin, TX, USA). The results were expressed as $\mathrm{pg} / \mathrm{ml}$.

\subsection{Statistical Analysis}

Statistical analysis was performed using GraphPad Prism 7.0 software (GraphPad, San Diego, CA, USA) and data were expressed as mean \pm standard error of the mean (SEM). Data are at least from three independent biological experiments. Three separated wells were processed for each independent experiment. To establish the distribution of the data, the test Shapiro Wilk was run. To assess the effect of every experimental condition compared to the other conditions, one-way ANOVA followed by Bonferroni's test (normal distribution) or the Kruskal-Wallis followed by Dunn's test (non-normal distribution) were performed. Significant differences were established at p-

\section{Results}

\subsection{Isolation and characterization of MVs produced by gut resident E. coli strains}

Previous studies by our group evidenced that MVs released by the probiotic EcN and the commensals ECOR63 and ECOR12 modulate in a strain-specific manner the expression of cytokines and tight junction proteins in intestinal epithelial cells (Fábrega et al., 2016; Alvarez et al., 2016; Cañas et al., 2018). To prove that secreted extracellular MVs are also a mechanism used by gut resident strains to specifically modulate human DCs and derived T cell responses, we isolated MVs from LB cultures 
326

327 from an $\mathrm{EcN}$ isogenic mutant unable to synthesise polysaccharide $\mathrm{K} 5$ were also purified to assess capsule-mediated effects on DCs. To evaluate strain-specific changes in vesicle size or structure, isolated MVs were examined by cryo-TEM (Fig. 1). As described for EcN vesicles (Pérez-Cruz et al., 2016), MVs from all E. coli strains had a spherical shape and a size between 20 and $200 \mathrm{~nm}$. Although size distribution varied according to the producer strain, most were in the range of $20-80 \mathrm{~nm}$, except for EcN:K5 that were enriched in MVs ranging from 80 to $110 \mathrm{~nm}$. In addition to the larger size, the bilayer surface of MVs from the K5 capsule-deficient mutant (EcN:K5) was thicker than that of the wild-type EcN vesicles. In all samples, common Gram-negative single-bilayer outer membrane vesicles predominated in all observed fields, but doublebilayer vesicles, named outer-inner membrane vesicles (Pérez-Cruz et al., 2015), were also observed occasionally (Fig. 1).

\subsection{MVs from gut resident E. coli strains elicit distinct pattern of cytokine secretion in stimulated human mo-DCs}

Considering that bacterial MVs serve as vehicles for cell-to-cell communication in the gut and that their effects are strain specific, we evaluated the ability of MVs isolated from gut resident E. coli strains to induce maturation of DCs in vitro. Human mo-DCs were exposed to MVs of each strain for $24 \mathrm{~h}$. In all assays, cell viability values above $95 \%$ were confirmed by flow cytometry analysis of both control and stimulated mo-DCs. In addition, viable cells were estimated by the MTT and trypan blue exclusion assays. Results showed no significant differences in MTT reduction levels between control and MV-treated mo-DCs (Figure S1).

Maturation of mo-DCs was assessed by flow cytometry analysis using the MODC differentiation inspector kit (Miltenyi Biotec). All MVs regardless of their origin induced high levels of the maturation surface marker CD83 compared to untreated moDCs, which confirms DC maturation. Consistently, vesicle-stimulated DCs showed reduced expression of CD209 (DC-SING) whereas CD14 expression remained low (Fig. 2).

Stimulation of mo-DCs with bacterial MVs also activated expression of cytokines to initiate specific immune responses. Cell culture supernatants from treated DCs were analysed after $24 \mathrm{~h}$ incubation for several secreted cytokines known to be 
359

360

361

362

363

364

365

366

367

368

369

370

371

372

373

374

375

376

377

378

379

380

381

382

383

384

385

386

387

388

389

390

391

related or to prime Th responses, namely INF- $\gamma$, IL-12 and IL-18 (Th1-related); IL-6, IL-23, TNF- $\alpha$ and IL-1 $\beta$ (Th17/Th22), and IL-10 and TGF- $\beta$ (Treg). In comparison with non-treated mo-DCs, MVs from all strains induced statistically significant secretion of all the cytokines assayed, although the observed profile differed greatly between strains (Fig. 3). MVs from the non-pathogenic E. coli B2 strains EcN (probiotic) and ECOR63 (commensal) elicited greater secretion of Th1 polarizing cytokines than MVs from ECOR12 MVs (group A) or the pathogenic ECOR53 (Fig. 3, panels A-C). In contrast, the highest expression levels of Treg-related cytokines, IL-10 and TGF- $\beta$, were achieved by treatment with ECOR12 MVs (Fig. 3, panels H,I). Vesicles from the probiotic $\mathrm{EcN}$ tend to induce greater secretion of Treg cytokines than MVs from other B2 strains although differences did not reach statistical significance (Fig. 3, panels H,I). All MVs enhanced secretion of Th17/Th22 priming cytokines, without significant differences between strains (Fig. 3, panels D-G). Considering K5mediated effects, the results showed that the cytokine secretion pattern elicited by EcN:K5 MVs only differed significantly from that of wild-type EcN MVs in the Th1driving cytokines, specifically INF- $\gamma$, IL-12 and IL-18 (Fig. 3, panels A-C).

Gene expression of cytokines commonly used as markers of DC activation was analysed by RT-qPCR at $6 \mathrm{~h}$ stimulation with MVs (Fig. 4). Results revealed higher mRNA levels for IL-6, TNF- $\alpha$, IL-10 and TGF- $\beta$ in stimulated DCs than in controls, regardless of the vesicle origin, except for TGF- $\beta$ expression (Fig. 4, panels A-C). The mRNA levels of TGF- $\beta$ in DCs incubated with ECOR53 MVs did not differ from control DCs (Fig. 4D). For each type of MVs, the mRNA expression profile was quite similar to that of the corresponding values of secreted cytokines (Fig. 3).

\subsection{The T cell responses triggered by MV-stimulated mo-DCs are strain specific}

We next evaluated the ability of MVs to induce DC modulation of $\mathrm{T}$ cell function and phenotype using vesicle-stimulated $\mathrm{DCs} / \mathrm{CD} 4^{+} \mathrm{T}$ cell in vitro co-cultures. As a control $\mathrm{CD}^{+} \mathrm{T}$ cells were co-incubated with non-treated DCs. After 3 days, secreted levels of IFN- $\gamma$, IL-17, IL-22, IL-4, IL-5, IL-13, IL-10 and TGF- $\beta$ were quantified (Fig. 5). DCs exposed to MVs isolated from gut beneficial bacteria induced higher levels of all cytokines than unstimulated DCs (Fig. 5, panels A-H), whereas DCs challenged with ECOR53 MVs failed to induce IL-10 and TGF- $\beta$ over control values (Fig. 5, panels G, H). All MVs activated Th-2 derived cytokines (IL-4, IL-5, IL-13) to 
392 similar levels (Fig. 5, panels D-F). A comparison of commensal and probiotic strains 393 revealed that ECOR12 MVs, a commensal that belongs to the phylogenetic group A, 394 triggered robust secretion of TGF- $\beta$ (Fig. $5 \mathrm{H}$ ) and lower expression of the inflammatory 395 cytokines INF- $\gamma$ (Th1 response) and IL-17 (Th17 response) than MVs from probiotic 396 EcN and other E. coli B2 strains (Fig. 5, panels A,B). No differences in the effects on 397 IL-22 secretion were observed between stimulated DCs, except for DCs exposed to 398 ECOR53 MVs that triggered significantly lower secreted IL-22 (Fig. 5C). A similar 399 pattern was observed for IL-10 secretion, although secreted values tended to be higher 400 in co-cultures containing DCs stimulated with EcN and ECOR12 MVs (Fig. 5G). The 401 ratio of TGF- $\beta / \mathrm{IL}-17$ revealed that cell responses induced by ECOR12 MVs are 402 predominantly directed towards TGF- $\beta$ secreting Treg cells, whereas probiotic EcN 403 induced compensated Treg/Th17 responses (Fig. 5I). The Th1 response was mainly 404 driven by E. coli strains belonging to group B2. Cytokine results were supported by 405 quantification of the Treg and Th17 subsets by cellular immunostaining for selective 406 markers after a 3-day co-incubation, specifically CD4/CD25 high/Foxp3 for Treg and 407 CD4/CD196 for Th17 (Fig. 6). Again, values of the Treg/Th17 ratio confirmed the 408 predominant Treg response induced by ECOR12 MVs. In the case of MVs from the 409 probiotic EcN, the results supported a balanced Treg/Th17 response, which was not 410 significantly affected by K5 deficiency. This analysis also showed that MVs of non411 probiotic B2 strains (ECOR63 and ECOR53) elicited lower Treg responses than EcN 412 MVs, although the differences did not reach statistical significance.

3.4. Effect of the epithelial layer on vesicle-mediated activation of immature $D C s$

416 Since in the gut DCs are in close contact and communicate with epithelial cells we 417 sought to examine the potential of MVs to indirectly stimulate DCs through the 418 intestinal epithelium layer. To this end, mo-DCs were incubated with supernatants 419 collected from the basolateral compartment of Caco-2 polarized monolayers exposed for $42024 \mathrm{~h}$ to MVs from the probiotic EcN and the commensal ECOR63 and ECOR12 strains. 421 Incubation of DCs with unstimulated Caco-2 conditioned medium was also performed 422 as a control of the basal epithelial - dendritic cell crosstalk. IL-10, TNF- $\alpha$ and IL-6 were 423 quantified after $24 \mathrm{~h}$ incubation of DCs with conditioned Caco-2 supernatants (Fig. 7A424 C, white bars). Levels of cytokines secreted by stimulated Caco-2 monolayers were 

435

measured in the collected supernatants before being used for DC stimulation (Fig. 7, 426 panels $\mathrm{A}-\mathrm{C}$, black bars). In the absence of vesicle-stimulation, Caco-2 conditioned 427 supernatants triggered secretion of TNF- $\alpha$ and IL-10 by immature mo-DCs (Fig. 7, 428 panels $\mathrm{B}-\mathrm{C}$, white bars) a finding compatible with the communication between 429 epithelial and immune cells by means of epithelial released factors. A two-fold increase 430 in the secreted values of both cytokines was observed in mo-DCs incubated with 431 supernatants from vesicle-exposed Caco-2 cell monolayers (Fig. 7, panels B-C, white 432 bars). In contrast, secretion of IL-6 by conditioned DCs was only detected when these 433 immune cells had been incubated with vesicle-exposed Caco-2 supernatants (Fig. 7, 434 panel A). In this undirect model of DC activation, cytokine values were substantially lower than those secreted by direct vesicle-stimulated DCs (Fig. 3, panels D, G, H).

\section{Discussion}

438 The probiotic $\mathrm{EcN}$ is the single active component of Mutaflor ${ }^{\circledR}$, a probiotic formula with proven effectiveness in the treatment of acute diarrhoea (Henker et al., 2007) and inflammatory intestinal disorders such as ulcerative colitis (Kruis et al., 2004; Kruis et al., 2012; Chibbar \& Dieleman, 2015). It has been widely reported that EcN positively modulates intestinal homeostasis and microbiota balance through several mechanisms that include: (i) modulation of the host immune response towards an anti-inflammatory balance (Trebichavsky et al., 2010), reinforcement of the intestinal epithelial barrier by strengthening tight junctions between intestinal epithelial cells (Ukena et al., 2007; Zyrek et al., 2007; Hering et al., 2014) and upregulation of antimicrobial factors such as microcins and $\beta$-defensin-2 (Schlee et al., 2007; Sassone-Corsi et al., 2016). This probiotic is in fact a gut beneficial bacterium that was originally isolated from a soldier who survived a severe outbreak of diarrhoea during the First World War. Like other components of the gut microbiota, probiotics use a wide range of signals to modulate the intestinal immune function. Due to the huge microbial population that inhabit the intestinal tract, host barrier and regulatory mechanisms are required to control bacteriahost reciprocal interactions, aimed at preventing aberrant responses and ensuring homeostasis. The first intestinal barrier that prevents direct contact between gut microbes and epithelial and immune cells is the mucus layer that covers the intestinal epithelium. Thus, gut microbiota-host crosstalk is mainly accomplished by secreted active mediators that can diffuse across the inner mucus layer. Now, bacterial 
extracellular vesicles are considered key players in bacteria-host communication, as they permit delivery of bacterial effectors into host cells without direct intercellular contact. MVs secreted by Gram-negative bacteria enclose a wide range of cargos, including numerous MAMPs such as LPS, lipoproteins, peptidoglycan, DNA and RNA that can engage host PRRs to initiate signalling cascades. Upon interaction with host epithelial or immune cells, bacterial MVs are internalized by endocytosis and intracellularly deliver the active compounds that elicit suitable responses (KaparakisLiaskos \& Ferrero, 2015).

Due to the importance of bacterial MVs in intercellular dialogue, research in this field was first focused on pathogen-derived MVs and infection diseases. Nowadays, the study of MVs secreted by gut microbiota and probiotics is an emerging topic (MolinTijeras et al., 2019). Our group was pioneer in the study of probiotic MVs, specifically vesicles from the Gram-negative probiotic $\mathrm{EcN}$. We focused on the proteomic characterization of EcN MVs and their immunomodulatory and barrier protective effects in intestinal epithelial cells (Aguilera et al., 2014; Fábrega et al., 2016; Alvarez et al., 2016; Cañas et al., 2018). We also reported that EcN MVs reduce inflammatory scores in a DSS-induced experimental colitis model in mice (Fábrega et al., 2017). Comparative studies revealed that MVs released by other intestinal E. coli also display some beneficial effects. Particularly, ECOR63 MVs reinforce tight junctions in intestinal cell monolayers (Alvarez et al., 2016) whereas ECOR12 lacks this activity but elicits immunomodulatory effects in intestinal epithelial cells and colonic explants (Fábrega et al., 2016; Cañas et al., 2018).

Here we have expanded our study to the effects mediated by MVs on DCs function, including vesicles from the probiotic $\mathrm{EcN}$ and from commensal E. coli strains isolated from faecal microbiota of healthy individuals and representing distinct populations. We showed that all the MVs that were analysed elicited maturation of moDCs but with distinct activation profile. No significant differences were observed in the expression of the general maturation marker CD83 between strains, whereas secreted levels of Treg and Th1-driving cytokines differed greatly, depending on the vesicle producer strain. The inflammatory Th1 response is crucial to fight against pathogens and resolve infection. In contrast, regulatory $\mathrm{T}$ cells are critical for sustained immune tolerance in the intestine. MVs from the B2 strains EcN and ECOR63, elicited higher secretion of INF- $\gamma$, IL-12 and IL-18 than MVs from the group A strain ECOR12. It is 
well-known that INF- $\gamma$ and IL-12 have potent effects on T cell differentiation into Th1 cells. Although IL-18 cannot induce Th1 cell development per se, it is considered a proinflammatory cytokine that potentiates type 1 responses by activating established Th1 cells to secrete IFN- $\gamma$ in the presence of IL-12 (Nakanishi, 2018). Therefore, activation of iDCs to secrete Th1 driving cytokines by EcN MVs may be one of the mechanisms exploited by this probiotic to protect against viral and bacterial enteric infections. This effect could be mediated, at least in part, by the K5 capsular polysaccharide. A similar EcN activation profile of Th1-driving cytokines was triggered by MVs from other intestinal B2 strains with beneficial traits such as ECOR63. Although it belongs to the same B2 group, ECOR53 MVs triggered significantly 501 reduced activation of INF- $\gamma$, IL-12 and IL-18. This difference may be associated with the potential pathogenic trait of ECOR53. The phylogenetic group B2 mainly encloses virulent strains that cause extra-intestinal infections. In fact, strain ECOR53, but not ECOR63, expresses functional haemolysin and produces strong cytotoxicity in macrophages (Lai et al., 1999). It is widely described that pathogenic bacteria have evolved multiple strategies to bypass the host cellular machinery and immune responses to ensure survival. One strategy is modulation of the host inflammatory response by expressing bacterial anti-inflammatory proteins that avoid pathogen clearance and help bacteria to persist in the host cells. Most of these proteins are adhesins or surface proteins, and hence could be enclosed in MVs (Sun, 2010). Our results suggest that an ECOR53 factor or protein that can modulate DC function towards a reduced Th1 response is secreted and delivered through MVs. Concerning secretion of Treg polarizing cytokines, IL-10 and specifically TGF- $\beta$, the results were clearly different. The highest activation was achieved by incubation with MVs isolated from ECOR12. The E. coli phylogenetic group A is predominant in human stool isolates and principally comprises commensal strains that lack virulence determinants. Thus, these "innocuous" E. coli strains may help to maintain gut homeostasis by enhancing innate immunity and controlling the Treg/Th17 balance towards an anti-inflammatory profile. Interestingly, EcN MVs elicited higher activation of TGF- $\beta$ and IL-10 secretion than other B2 strains, a fact that is consistent with the probiotic trait of this strain. Collectively, these data indicate that the probiotic EcN modulated DCs towards better balanced Treg/Th17 responses than non-probiotic B2 strains. Regarding the expression of Th17 and Th22driving cytokines (IL-6, IL1- $\beta$, IL-23 and TNF- $\alpha$ ) (Eyerich et al., 2017), our results showed that all MVs could activate these cytokines to similar levels, without significant 
525 differences between the non-pathogenic E. coli strains. However, secreted levels of IL52623 (Th17 expansion) and TNF- $\alpha$ (Th22-differentiation) were significantly lower in DCs 527 stimulated with ECOR53 MVs. Th17 and Th22 cell lineages share some functional 528 features but the corresponding secreted cytokines specifically differ in some activities. 529 IL-17 is mostly a pro-inflammatory cytokine involved in protection against extracellular 530 bacterial infections, whereas IL-22 has high potential to protect tissue integrity and help 531 tissue regeneration (Eyerich et al., 2017).

532 Subsequent co-incubation of vesicle-stimulated DCs and allogenic CD4 ${ }^{+} \mathrm{T}$ cells 533 proved the ability of activated DCs to drive complex Th responses that were consistent 534 with their secreted cytokine profile. MVs from all strains analysed triggered similar Th2 535 responses. Except for ECOR53, all MVs activated IL-10 secreting $\mathrm{T}$ cells and 536 promoted a strong Th22 response. In addition, MVs from the probiotic EcN induced an 537 intricate response involving INF- $\gamma$ secreting Th1 cells, IL-17 secreting Th17 cells and 538 TGF- $\beta$ secreting FoxP3 ${ }^{+}$Treg cells. A similar response was activated by ECOR63 MVs. 539 Nonetheless, levels of secreted pro-inflammatory INF- $\gamma$ were higher than those induced 540 by EcN MVs and IL-10 and TGF- $\beta$ levels tended to be lower. Consistently, values of 541 the IL-10/INF- $\gamma$ ratio revealed that the Th response induced by ECOR63 MVs has a 542 stronger pro-inflammatory profile than those driven by EcN or ECOR12 MV. No 543 significant differences were observed in the Th response triggered by DCs stimulated 544 with EcN K5-deficient MVs versus wild-type EcN MVs except for the significantly 545 lower secretion of IFN- $\gamma$. These data correlate with the profile of DCs stimulated with 546 these MVs and point to K5 capsule as a modulator factor of Th1-related cytokines. 547 Concerning strain ECOR12, the MVs also induced a combined Th1-Th2-Th17-Th22548 Treg response, but the overall outcome is dominated by high levels of TGF- $\beta$, the 549 unique cytokine secreted by Treg cells. In this case, levels of Th1 and Th17-secreted 550 cytokines were lower than those elicited by EcN MVs. Flow cytometry analysis of Th17 551 and Treg markers confirmed the T cell subset profile induced by the different MVs: (i) a 552 similar increase in the percentage of Th17 cells $\left(\mathrm{CD}^{+} \mathrm{CD} 196^{+}\right)$induced by non553 pathogenic MVs and a reduced proportion in the case of ECOR53 MVs, and (ii) 554 ECOR12 promoted the highest increase in the Foxp $3^{+}$Treg population, followed 555 sequentially by $\mathrm{EcN}$ and ECOR63. MVs from ECOR53 failed to increase this $\mathrm{T}$ cell 556 population over the control (iDCS). 
As stated above, DCs preserve intestinal homeostasis by tuning the host immune system in response to gut microbes. However, in the normal gut very few bacteria can cross the inner mucin layer to reach DCs associated with the intestinal epithelium. In the present study, we have shown that the immune modulating properties of the probiotic $\mathrm{EcN}$ and commensal strains are mediated by secreted extracellular vesicles, which can go through the mucin layer (Cañas et al. 2016) and be easily sensed by M cells or phagocyted by DCs. Microbiota-derived vesicles also interact with the intestinal epithelium and modulate its functions. As stated above, previous studies of our group proved that microbiota vesicles are internalized in intestinal epithelial cells and modulate the immune and defence responses in a strain-specific manner. The intestinal epithelium layer is a key regulator of barrier function and immune homeostasis. Among the diverse functions, intestinal epithelial cells sense the gut microbial population, respond to microbial stimuli and coordinate appropriate immune responses. It has been shown that, upon contact with bacteria, intestinal epithelial cells release mediators that modulate immune cells in the lamina propria, favouring a tolerant and permissive environment for colonization by commensal bacteria (reviewed in Peterson \& Artis, 2014). Many studies in this field have been performed by incubating intestinal epithelial cells with bacterial suspensions. Here we have shown that intestinal epithelial monolayers exposed to MVs from intestinal E. coli strains can integrate and transmit signals to DCs. Activation of DCs through the epithelial barrier resulted in lower secretion of IL-6, TNF- $\alpha$ and IL-10 than direct activation by MVs, thus suggesting that microbiota-derived MVs modulate the epithelial-dendritic cell crosstalk and contribute to DC training towards low responsiveness against commensal bacteria.

Due to the health-promoting capacities of probiotics, their inclusion in processed foods and potential clinical applications are receiving considerable attention in our society. Nonetheless, the translation of probiotics or microbiota-based treatments to human health depends on basic knowledge of the molecular mechanisms involved in their interaction with the host. It should also be considered that live probiotics may have putative adverse effects or cause invasive infections in immunocompromised patients. In this context, probiotic-derived MVs could be explored as a new and safe (bacteriafree) strategy targeting gut microbiota balance or intestinal inflammatory disorders.

\section{Conclusion}


590 Gut beneficial microbes modulate the innate immune system and preserve intestinal 591 homeostasis by facilitating quick responses against pathogens and ensuring tolerance to 592 symbiont bacteria. Here we provide evidence that MVs represent a mechanism used by 593 probiotics and commensal strains to steadily prime the innate immune system, 594 activating DCs in a strain-specific manner. MVs from the probiotic EcN drive complex 595 Th responses: Th2 (repair response against tissue damaging nematodes), Th22 (tissue 596 protection), Th1 (pro-inflammatory, protective immunity to pathogens), Th17 (pro597 inflammatory, antimicrobial response) and Treg (immune tolerance). Besides these 598 balanced immunomodulatory activities, EcN MVs reinforce intestinal epithelial tight 599 junctions. Thus, secreted MVs mediate the beneficial effects of this probiotic. Other 600 commensal E. coli strains from the group B2 such as ECOR63 also have barrier 601 strengthening activity and elicit similar Th responses but with a stronger pro602 inflammatory profile. Concerning the commensal strain ECOR12, secreted MVs mainly 603 contribute to maintaining host tolerance against gut microbes by increasing the 604 Treg/Th17 balance and protecting gut integrity by activating the Th22 response. In 605 contrast, ECOR12 MVs do not trigger appropriate Th1 responses against pathogens nor 606 do they reinforce tight junctions.

607

608

609 Funding

610 This study was supported by Grant AGL2016-79113-R, co-financed with European 611 Commission ERDF funds, from the Ministerio de Economía, Industria y 612 Competitividad Spain (AEI/FEDER, UE) to LB. N. Diaz-Garrido acknowledges her 613 doctoral fellowship from CONICYT - Becas Chile. We acknowledge financial support 614 from Universitat de Barcelona for publication in open access.

615

\section{Acknowledgments}

617 We acknowledge laboratories Ardeypharm and I.S. Roberts for providing the probitic $618 \mathrm{EcN}$ and the EcN K5-deficient mutant, respectively. We thank F.J Pérez-Cano 619 (Professor of Physiology, University of Barcelona) for helpful discussion and J. Comas 620 (staff of the Scientific and Technological Centres of the University of Barcelona, CCiT- 
621 UB) for technical support in flow cytometry analysis. We acknowledge Lucille Banham

622 for assistance in preparing the English manuscript.

623

624 Conflict of Interest Statement

625 The authors declare that the research was conducted in the absence of any commercial

626 or financial relationships that could be construed as a potential conflict of interest.

627

628

629 Ethics statement

630 Use of anonymous, non-identifiable human samples was approved by the Bioethics

631 Committee of the University of Barcelona (Institutional Review Board:1R800003099).

632

633 Author contributions

634 LB and JB conceived of the study with the contribution of RG, MJF and NDG in 635 experimental design. LB and JB wrote the manuscript and supervised the work. RG, RV 636 and NDG carried out data interpretation and statistical analysis. NDG, MJF and RV 637 executed the experimental work. All authors revised, read and approved the final 638 manuscript.

639 
640

641

642

643

644

645

646

647

648

649

650

651

652

653

654

655

656

657

658

659

660

661

662

663

664

665

666

667

668

669

670

\section{References}

Aguilera, L., Toloza, L., Giménez, R., Odena, A., Oliveira, E., Aguilar, J., Badia, J., \& Baldomà, L. (2014). Proteomic analysis of outer membrane vesicles from the probiotic strain Escherichia coli Nissle 1917. Proteomics, 14, 222-229. https://doi.org/10.1002/pmic. 201300328.

Alvarez, C-S., Badia, J., Bosch, M., Giménez, R., \& Baldomà, L. (2016). Outer membrane vesicles and soluble factors released by probiotic Escherichia coli Nissle 1917 and commensal ECOR63 enhance barrier function by regulating expression of tight junction proteins in intestinal epithelial cells. Frontiers in Microbiology, 7, 1981. https://doi.org/10.3389/fmicb.2016.01981.

Baothman, O. A., Zamzami, M. A., Taher. I., Abubaker, J., \& Abu-Farha, M. (2016). The role of gut microbiota in the development of obesity and diabetes. Lipids in Health and Disease, 15, 108. https://doi.org/10.1186/s12944-016-0278-4

Belkaid, Y., \& Hand, T. (2014) Role of microbiota in immunity and inflammation. Cell, 157(1), 121-141. https://doi.org/10.1016/j.cell.2014.03.011.

Cañas, M. A., Giménez, R., Fábrega, M. J., Toloza, L., Baldomà, L., \& Badia, J. (2016). Outer membrane vesicles from the probiotic Escherichia coli Nissle 1917 and the commensal ECOR12 enter intestinal epithelial cells via clathrin-dependent endocytosis and elicit differential effects on DNA damage. PLoS One, 11(8), e0160374. https://doi.org/10.1371/journal.pone.0160374.

Cañas, M. A., Fábrega, M.J., Giménez, R., Badia, J., \& Baldomà, L. (2018). Outer membrane vesicles from probiotic and commensal Escherichia coli activate Nod1mediated immune responses in intestinal epithelial cells. Frontiers in Microbiology, 9, 98. https://doi.org/10.3389/fmicb.2018.00498.

Chibbar, R., \& Dieleman, I.A. (2015). Probiotics in the management of ulcerative colitis. Journal of Clinical Gastroeneterology, 49, S50-S55. https://doi.org/10.1097/MCG.0000000000000368.

Eyerich, K., Dimartino, V., \& Cavani, A. (2017). IL-17 and IL-22 in immunity: driving protection and pathology. European journal of Immunology, 47(4), 607-614. https://doi.org/10.1002/eji.201646723. 
671 Fábrega, M. J., Aguilera, L., Giménez, R., Varela, E., Cañas, M. A., Antolín, M., Badía,

J., \& Baldomà, L. (2016). Activation of immune and defense responses in the intestinal mucosa by outer membrane vesicles of commensal and probiotic Escherichia coli strains. Frontiers in Microbiology, 7, 705. https://doi.org/10.3389/fmicb.2016.00705.

Fábrega, M. J., Rodríguez-Nogales, A., Garrido-Mesa, J., Algieri, F., Badía, J., Giménez, R., Gálvez, J., \& Baldomà, L. (2017). Intestinal anti-inflammatory effects of outer membrane vesicles from Escherichia coli Nissle 1917 in DSSexperimental colitis in mice. Frontiers in Microbiology, 8, 1274. https://doi.org/10.3389/fmicb. 2017.01274.

Gopalakrishnan,V., Helmink, B. A., Spencer, C. N., Reuben, A., \& Wargo, J. A. (2018). The influence of the gut microbiome on cancer, immunity and cancer immunotherapy. Cancer Cell. 33, 570-580. https://doi.org/10.1016/j.ccell. 2018.03.015.

Hafez, M., Hayes, K., Goldrick, M., Warhurst, G., Grencis, R., \& Roberts I. S. (2009). The K5 capsule of Escherichia coli strain Nissle 1917 is important in mediating interactions with intestinal epithelial cells and chemokine induction. Infection \& Immunity, 77(7), 2995-3003. https://doi.org/10.1128/IAI.00040-09.

Hafez, M., Hayes, K., Goldrick, M., Grencis, R. K., \& Roberts, I. S. (2010). The K5 capsule of Escherichia coli strain Nissle 1917 is important in stimulating expression of Toll-like receptor 5, CD14, MyD88, and TRIF together with the induction of interleukin- 8 expression via the mitogen-activated protein kinase pathway in epithelial cells. Infection \& Immunity, 78(5), 2153-2162. https://doi.org/10.1128/IAI.01406-09.

Hafez, M. M. (2012). Upregulation of intestinal mucin expression by the probiotic bacterium E. coli Nissle 1917. Probiotics and Antimicrobrial Proteins, 4(2), 67-77. https://doi.org/10.1007/s12602-012-9092-0.

Henker, J., Laass, M., Blokhin, B.M., Bolbot, Y. K., Maydannik, V. G., Elze, M., Wolff, C., \& Schulze J. (2007). The probiotic Escherichia coli strain Nissle 1917 $(\mathrm{EcN})$ stops acute diarrhoea in infants and toddlers. European Journal of Pediatrics, 166, 311-318. https://doi.org/10.1007/s00431-007-0419-x. 
702

Höpken, U. E., Lehmann, I., Droese, J., Lipp, M, Schüler, T., \& Rehm, A. (2005). The ratio between dendritic cells and $\mathrm{T}$ cells determines the outcome of their encounter. Proliferation versus deletion. European Journal of Immunology, 35,2851-2863. https://doi.org/10.1002/eji.20052698

Jandhyala, S. M., Talukdar, R., Subramanyam, C., Vuyyuru, H., Sasikala, M., \& Reddy, N. D. (2015). Role of the normal gut microbiota. World Journal of Gastroenterology, 21(29), 8787-8803. https://doi.org/10.3748/wjg. v21.i29.8787.

Jia, W., Li, H., Zhao, L., \& Nicholson, J. K. (2008). Gut microbiota: a potential new territory for drug targeting. Nature Reviews Drug Discovery, 7, 123-129. https://doi.org/10.1038/nrd2505.

Kaparakis-Liaskos, M., \& Ferrero, R. L. (2015). Immune modulation by bacterial outer membrane vesicles. Nature Reviews Immunology, 15(6), 375-387. https://doi.org/10.1038/nri3837.

Kruis, W., Frič, P., Pokrotnieks, J., Lukáš, M., Fixa, B., Kaščák, M., Kamm, MA., Weismueller, J., Beglinger, C., Stolte, M., Wolff, C., \& Schulze, J. (2004). Maintaining remission of ulcerative colitis with the probiotic Escherichia coli Nissle 1917 is as effective as with standard mesalazine. Gut, 53, 1617-23. https://doi.org/10.1136/gut.2003.03774720.

Kruis, W., Chrubasik, S., Boehm, S., Stange, C., \& Schulze, J. (2012). A double-blind placebo-controlled trial to study therapeutic effects of probiotic Escherichia coli Nissle 1917 in subgroups of patients with irritable bowel syndrome. International Journal of Colorectal Disease, 27(4), 467-74. https://doi.org/10.1007/s00384-0111363-9.

Lai, X.H., Wang, S.Y., \& Uhlin, B. E. (1999). Expression of cytotoxicity by potential pathogens in the standard Escherichia coli collection of reference (ECOR) strains. Microbiology 145, 3295-3303. doi: 10.1099/00221287-145-11-3295.

Livak, K. J., \& Schmittgen, T. D. (2001). Analysis of relative gene expression data using real-time quantitative PCR and the $2^{-\Delta \Delta C}$ T method. Methods, 25, 402-408. https://doi.org/10.1006/meth.2001.1262.

López, P., González-Rodríguez, I., Sánchez, B., Gueimonde, M., Margolles, A., \& Suárez, A. (2012). Treg-inducing membrane vesicles from Bifidobacterium bifidum 
LMG13195 as potential adjuvants in immunotherapy. Vaccine, 30(5), 825-829. https://doi.org/10.1016/j.vaccine.2011.11.115.

Maguire, M., \& Maguire, G. (2018). Gut dysbiosis, leaky gut, and intestinal epithelial proliferation in neurological disorders: towards the development of a new therapeutic using amino acids, prebiotics, probiotics, and postbiotics. Reviews in the Neurosciences, 30(2), 179-201. https://doi.org/10.1515/revneuro-2018-0024.

Marin, I. A., Goertz, J. E., Ren, T., Rich, S. S., Onengut-Gumuscu, S., Farber, E., Wu, M., Overall, C. C., Kipnis, J., \& Gaultier, A. (2017). Microbiota alteration is associated with the development of stress-induced despair behavior. Scientific Reports, 7, 43859. https://doi.org/10. 1038/srep43859

Nakanishi, K. (2018). Unique action of interleukin-18 on T cells and other immune cells. Frontiers in Immunology, 9, 763. https://doi.org/10.3389/fimmu.2018.00763.

Nissle, A. (1916). Über die Grundlagen einer neuen ursächlichen Bekämpfung der pathologischen Darmflora. Deutsche medizinische Wochenschrift, 42,1181-1184.

Nzakizwanayo, J., Kumar, S., Ogilvie, L. A., Patel, B. A., Dedi, C., Macfarlane, W. M., \& Jones, B. V. (2015). Disruption of Escherichia coli Nissle 1917 K5 capsule biosynthesis, through loss of distinct $k f i$ genes, modulates interaction with intestinal epithelial cells and impact on cell health. PLoS One, 10(3), e0120430. https://doi.org/10.1371/journal.pone.0120430.

Ochman, H., \& Selander, R. K. (1984). Standard reference strains of Escherichia coli from natural populations. Journal of Bacteriology, 157, 690-693.

Olier, M., Marcq, I., Salvador-Cartier, C., Secher, T., Dobrindt, U., Boury, M., Bacquié, V., Pénary, M., Gaultier, E., Nougayrède, J. P., Fioramonti, J., \& Oswald, E. (2012). Genotoxicity of Escherichia coli Nissle 1917 strain cannot be dissociated from its probiotic activity. Gut Microbes, 3, 501-509. https://doi.org/10.4161/ gmic. 21737.

Perez-Cruz, C., Delgado, L., López-Iglesias, C., \& Mercade, E. (2015). Outer-inner membrane vesicles naturally secreted by gram-negative pathogenic bacteria. PLoS One, 10(1), e0116896. https://doi.org/10.1371/journal.pone. 0116896.

Pérez-Cruz, C., Cañas, M. A., Giménez, R., Badia, J., Mercade, E., Baldomà, L., \& Aguilera, L. (2016). Membrane vesicles released by a hypervesiculating 
Escherichia coli Nissle 1917 tolR mutant are highly heterogeneous and show reduced capacity for epithelial cell interaction and entry. PLoS One, 11(12):e0169186. https://doi.org/10.1371/journal.pone.0169186.

Peterson, L. W., \& Artis D. (2014). Intestinal epithelial cells: regulators of barrier function and immune homeostasis. Nature Reviews Immunology, 14, 141-153. https://doi.org/ 10.1038/nri3608.

Sabharwal, H., Cichon, C., Ölschläger, T. A, Sonnenborn. U., \& Schmidt, M. A. (2016). Interleukin-8, CXCL1, and microRNA miR-146a responses to probiotic Escherichia coli Nissle 1917 and enteropathogenic E. coli in human intestinal epithelial T84 and monocytic THP-1 cells after apical or basolateral infection. Infection \& Immunity, 84(9), 2482-2492. https://doi.org/10.1128/IAI.00402-16.

Shanahan, F. (2011). The gut microbiota in 2011: Translating the microbiota to medicine. Nature Reviews Gastroenterology \& Hepatology, 9(2), 72-74. https://doi.org/10.1038/nrgastro.2011.250.

Shen, Y., Giordano Torchia, M. L., Lawson, G.W., Karp, C. L., Ashwell, J.D., \& Mazmanian, S. K. (2012). Outer membrane vesicles of a human commensal mediate immune regulation and disease protection. Cell Host \& Microbe, 12(4), 509-520. https://doi.org/10.1016/j.chom.2012.08.004.

Sonnenborn, U., \& Schulze, J. (2009). The non-pathogenic Escherichia coli strain Nissle 1917 - features of a versatile probiotic. Microbial Ecology in Health and Disease, 21, 122-158, https://doi.org/10.3109/08910600903444267.

Sun, J. (2010). Pathogenic bacterial proteins and their anti-inflammatory effects in the eukaryotic host. Anti-inflammatory and Anti-allergy Agents in Medical Chemistry, 8(3): 214-227. https://doi.org/10.2174/187152309789151986.

Thursby, E., \& Juge, N. (2017). Introduction to the human gut microbiota. Biochemical Journal, 474 (11), 1823-1836. https://doi.org/10.1042/BCJ20160510.

Toloza, L., Gimenez, R., Fabrega, M. J., Alvarez, C. S., Aguilera, L., Cañas, M. A., Martín-Venegas, R., Badia, J., \& Baldomà, L. (2015). The secreted autotransporter toxin (Sat) does not act as a virulence factor in the probiotic Escherichia coli strain Nissle 1917. BMC Microbiology, 15, 250. https://doi.org/10.1186/s12866-0150591-5. 
795 Trebichavsky, I., Splichal, I., Rada, V., \& Splichalova, A. (2010). Modulation of

796 natural immunity in the gut by Escherichia coli Nissle 1917. Nutrition Reviews 68,

797 459-64.

798 Vejborg, R. M., Friis, C., Hancock, V., Schembri, M. A., \& Klemm, P. (2010). A

799 virulent parent with probiotic progeny: comparative genomics of Escherichia coli strains CFT073, Nissle 1917 and ABU 83972. Molecular Genetics and Genomics, 283(5), 469-484. https://doi.org/10.1007/s00438-010-0532-9.

802 
804 Fig. 1. Cryo-TEM observation of isolated MVs from the indicated E. coli strains. 805 Representative images of plunge-frozen MVs are shown. All samples were enriched in 806 the conventional outer membrane vesicles originating from the outer membrane of Gram807 negative bacteria, but low-abundant outer-inner membrane vesicles (O-IMVs) were also 808 observed. The image in the last panel shows an O-IMVS, in which the double-bilayer 809 membrane structure is well defined. Scale bars: $200 \mathrm{~nm}$.

810 Fig. 2. Maturation of DCs treated with MVs from the indicated probiotic and 811 commensal E. coli strains. Expression of CD14, CD83 and CD209 in mo-DCs exposed 812 to bacterial MVs $(10 \mu \mathrm{g} / \mathrm{ml})$ for $24 \mathrm{~h}$ was analysed by flow cytometry. Untreated mo813 DCs were kept in DC medium as a control of immature DCs. (A) A representative panel 814 of flow cytometry histograms is shown, where values of untreated mo-DCs are 815 indicated in red and those from vesicle-treated DCs in light blue. Overlapping areas are 816 visualized in dark blue. (B) Quantification of CD14, CD209 and CD83 expression. The 817 graph shows fold-changes in the mean fluorescence intensity (mean \pm SEM) of the 818 respective marker expression compared to control DCs (dot line). Data are from three independent biological experiments (at least three donors) performed in triplicate. 820 Statistical differences were evaluated by one-way ANOVA followed by Bonferroni's 821 test. ${ }^{*} p<0.05$ versus control DCs, \#p<0.05 versus DCs stimulated with EcN MVs.

822 Fig. 3. DC modulation by MVs from probiotic and gut resident E. coli strains. (A-I) 823 Levels of INF- $\gamma$, IL-12, IL-18, IL-6, IL-23, IL-1 $\beta$, TNF- $\alpha$, IL-10 and TGF- $\beta$ secreted by 824 mo-DCs following $24 \mathrm{~h}$ incubation with MVs $(10 \mu \mathrm{g} / \mathrm{ml})$ of the indicated strains. 825 Untreated mo-DCs were kept in DC medium as a control (black bars). Data (mean \pm 826 SEM) are from three independent biological experiments (at least three donors) 827 performed in triplicate. Statistical differences were evaluated by one-way ANOVA 828 followed by Bonferroni's test (normal distribution) or the Kruskal-Wallis followed by 829 Dunn's test (non-normal distribution). ${ }^{*} p<0.05$ versus control DCs, $\# p<0.05$ versus 830 DCs stimulated with EcN MVs.

832 Fig. 4. Gene expression levels of selected cytokines by MV-stimulated DCs. Immature 833 mo-DCs were challenged for $6 \mathrm{~h}$ with MVs $(10 \mu \mathrm{g} / \mathrm{ml})$ of the indicated strains. (A-D) 834 Relative mRNA levels of IL-6, TNF- $\alpha$, IL-10 and TGF- $\beta$ were measured by RT-qPCR, 835 using HPRT-1 as the reference gene. Data (mean \pm SEM) are from three independent 
836 biological experiments (at least three donors) performed in triplicate and are presented 837 as fold-change compared to untreated control cells. Statistical differences were 838 evaluated by one-way ANOVA followed by Bonferroni's test. ${ }^{*} p<0.05$ versus control 839 DCs, \#p<0.05 versus DCs stimulated with EcN MVs.

840

841 Fig. 5. MV-stimulated DCs modulate $\mathrm{CD}^{+} \mathrm{T}$ cell responses in a strain-specific manner. 842 (A-H) Quantification of secreted INF- $\gamma$, IL-17, IL-22, IL-4, IL-5, IL-13, IL-10, TGF- $\beta$ 843 and TNF- $\alpha$ by 3-day cocultures of MV-stimulated DCs and allogenic CD4+ T cells at a 844 DC:T cell ratio of 1:5. Parallel cocultures with untreated iDCs were processed as a 845 control. (I) Ratio of TGF- $\beta / \mathrm{IL}-17$ values. Data (mean \pm SEM) are from three 846 independent biological experiments performed in triplicate. Statistical differences were 847 evaluated by one-way ANOVA followed by Bonferroni's test (normal distribution) or 848 the Kruskal-Wallis followed by Dunn's test (non-normal distribution). ${ }^{*} p<0.05$ versus control DCs, \#p<0.05 versus DCs stimulated with EcN MVs.

850

851 Fig. 6. Quantification of the Th17 and Treg subsets induced by MV-stimulated DCs. 852 (A-B) Flow cytometric analysis of $\mathrm{CD}^{+} \mathrm{T}$ cells co-incubated for 3 days with untreated 853 mo-DCs (iDC) or mo-DCs primed with MVs of the indicated strains $(10 \mu \mathrm{g} / \mathrm{ml})$ at a 854 DC:T cell ratio of 1:5 with specific markers for: (A) Th17 population $\left(\mathrm{CD} 4^{+} \mathrm{CD} 196^{+}\right)$ 855 and (B) Treg $\left(\mathrm{CD} 25^{+}(\mathrm{High}) \mathrm{FoxP}^{+}\right)$population. Representative dot plots of $\mathrm{CD} 4^{+}$cells 856 co-incubated with iDCs (control) or with EcN MV-stimulated DCS are shown for each 857 cell subset analysis. The percentage of positive cells is indicated in each quadrant or 858 box. (C) Data (mean \pm SEM) from three-independent experiments performed in 859 triplicate are expressed as fold-change increase in the percentage of positive $\mathrm{T}$ cells for 860 Th17 or Treg specific markers induced by MV-stimulated DCs with respect control 861 iDCs induced values (set as 1, dot line). Statistical differences were evaluated by one862 way ANOVA followed by Bonferroni's test (normal distribution) $* p<0.05$ versus 863 control DCs.

Fig. 7. Intestinal epithelial cells modulate cytokine production from DCs. Mo-DCs were 866 incubated for $24 \mathrm{~h}$ with supernatants collected from the basolateral membrane of polarized Caco-2 monolayers challenged with MVs $(10 \mu \mathrm{g} / \mathrm{ml})$ of the indicated strains. Untreated Caco-2 cell supernatants were used in parallel for comparison. (A) IL-6, (B) 
869 TNF- $\alpha$ and (C) IL-10 secreted levels were quantified in Caco-2 basolateral supernatants 870 (black bars) and in conditioned DCs cultures (white bars). Data (mean \pm SEM) are from 871 three independent biological experiments performed in duplicate. Statistical differences 872 were evaluated by one-way ANOVA followed by Bonferroni's test (normal 873 distribution) *p $<0.05$ versus DCs incubated with untreated Caco-2 supernatants. 
Table 1. Primers used in this study

\begin{tabular}{lll}
\hline Gene & Sequence & $\begin{array}{l}\text { Gene accession } \\
\text { number }\end{array}$ \\
\hline IL-6 & F- AGCCACTCACCTCTTCAGAAC & NM_000600.4 \\
TNF- $\boldsymbol{\alpha}$ & F- CTCCTCTTTGCTGCTTTCACAC & \\
& R- AGATGATCTGACTGCCTGGG & NM_000594.2 \\
IL-10 & F- GCCTAACATGCTTCGAGATC & NM_000572.2 \\
TGF- $\boldsymbol{\beta}$ & R- TGATGTCTGGGTCTTGGTTC & \\
& F- CGAGAAGCGGTACCTGAAC & NM_000660.6 \\
HPRT-1 & R- TGAGGTATCGCCAGGAATTGT & \\
& F-CCTGGCGTCGTGATTAGTGAT & NM_000194 \\
\hline
\end{tabular}


Click here to download high resolution image

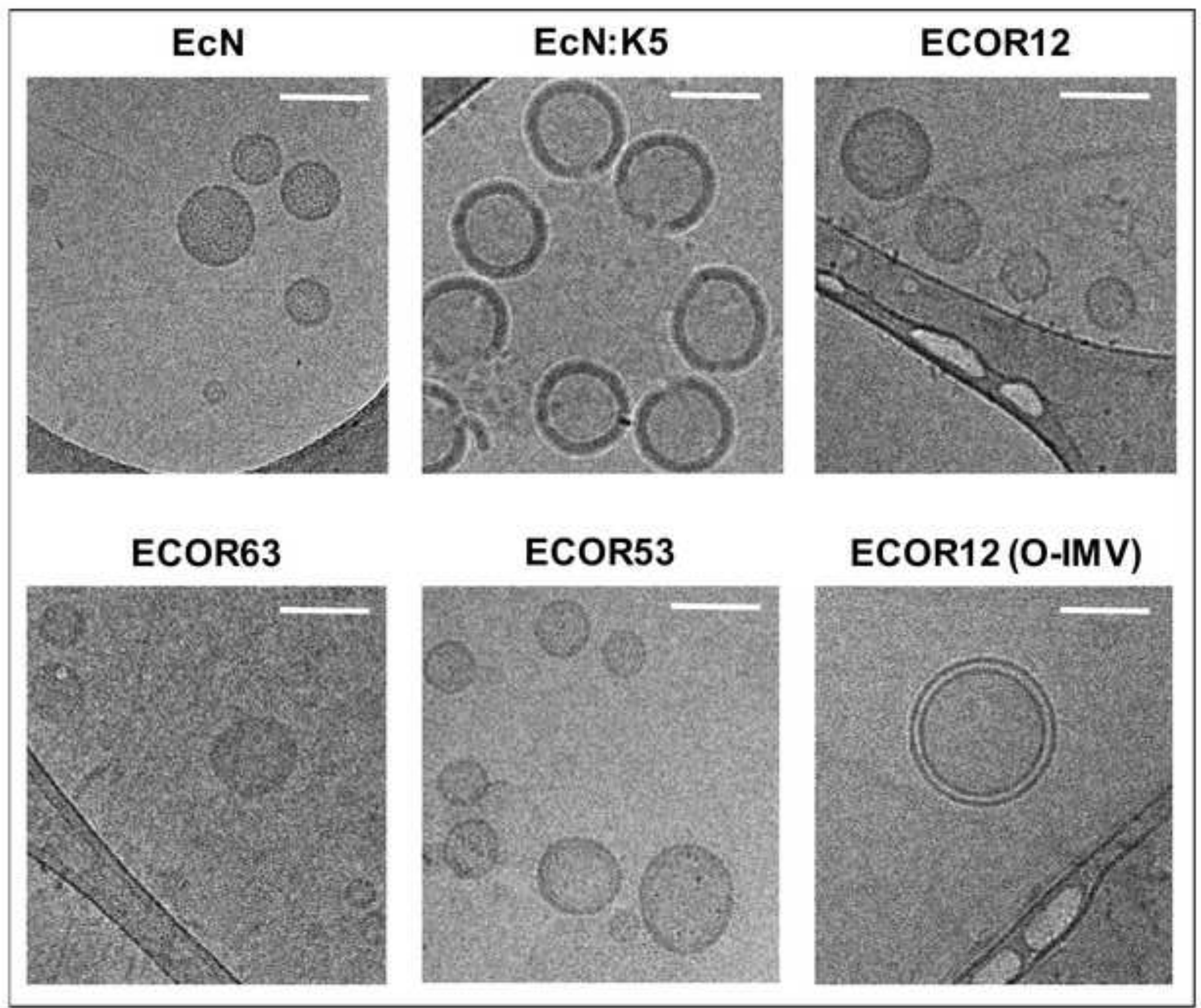




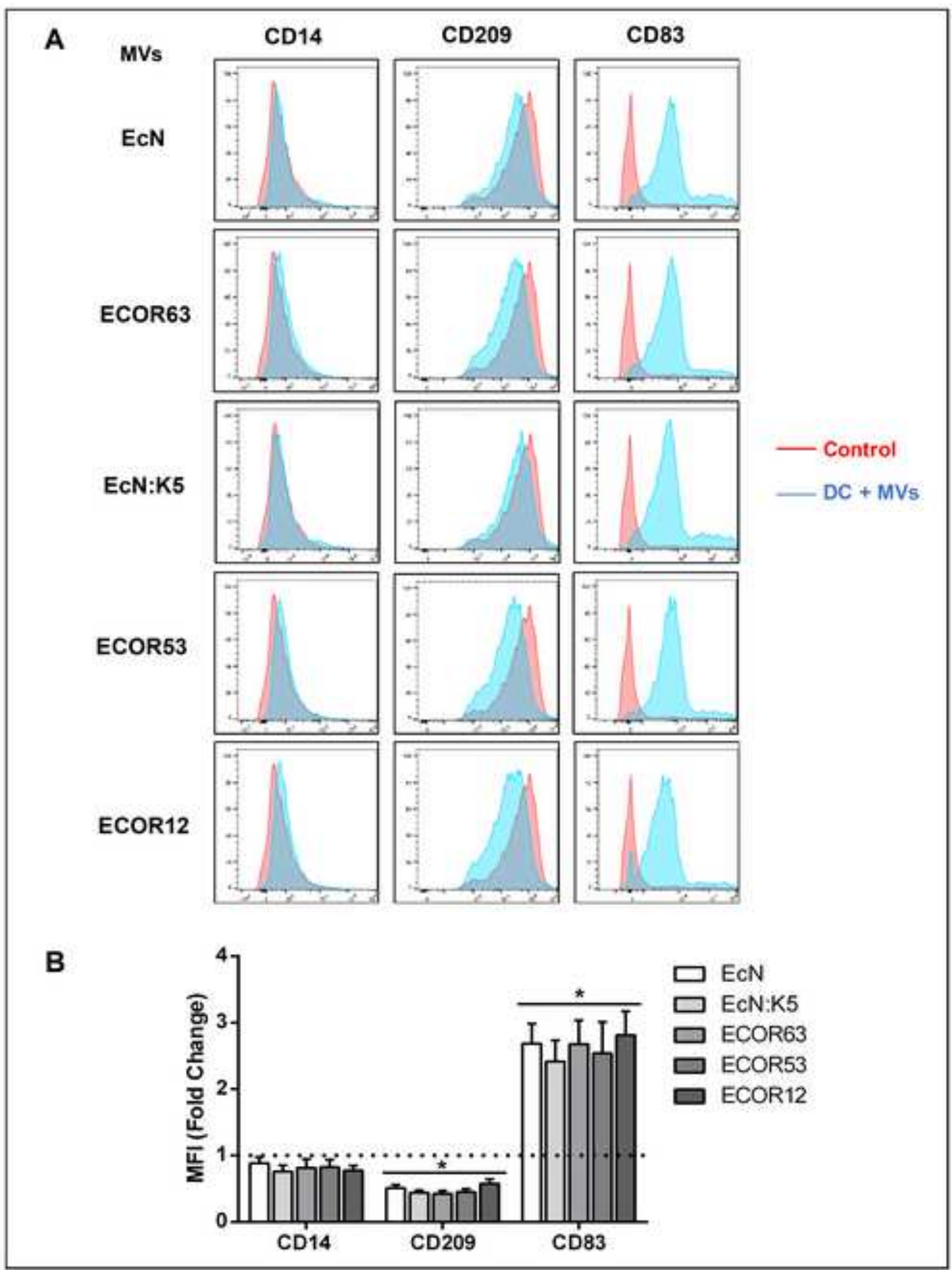


A

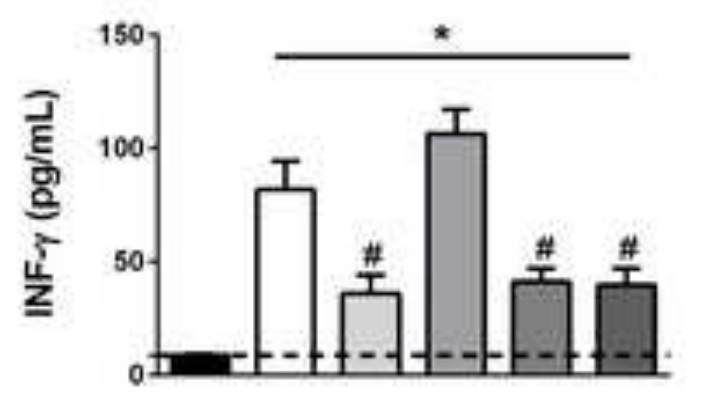

D

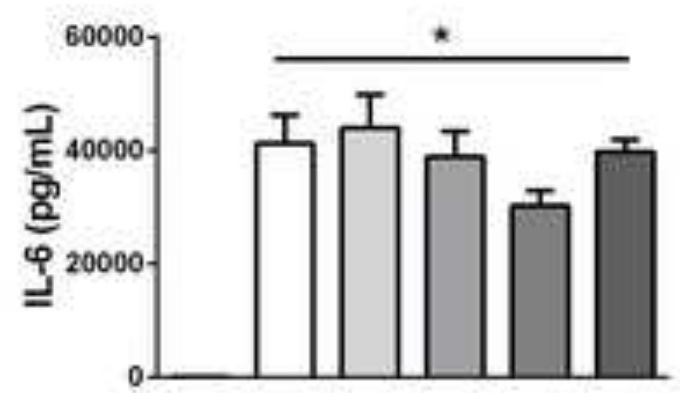

G

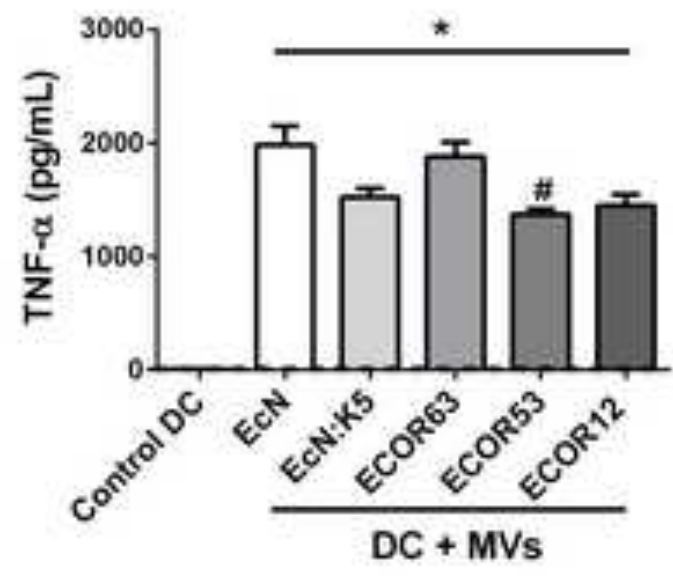

B

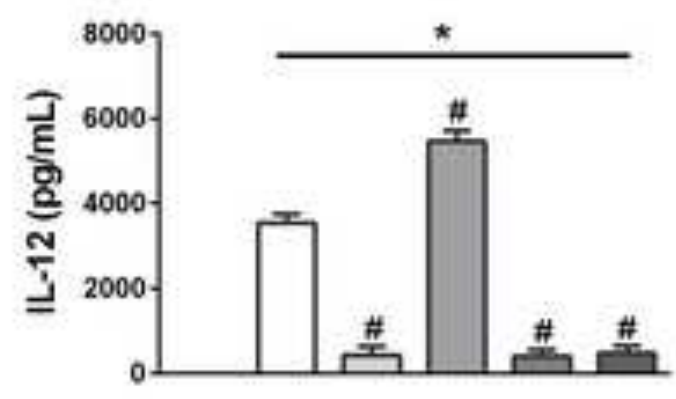

E

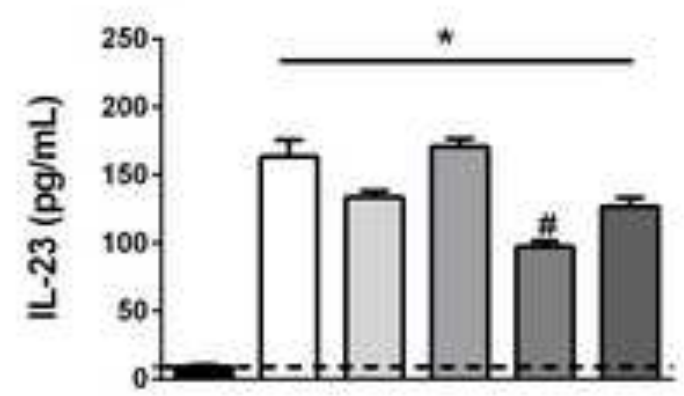

H

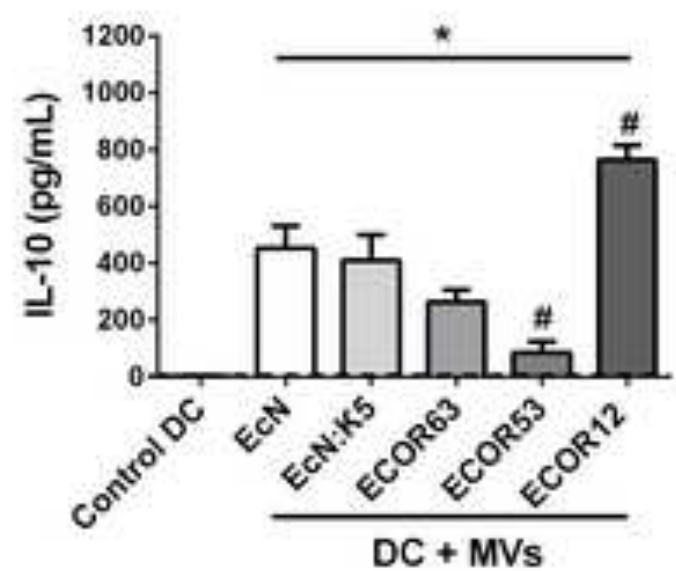

C

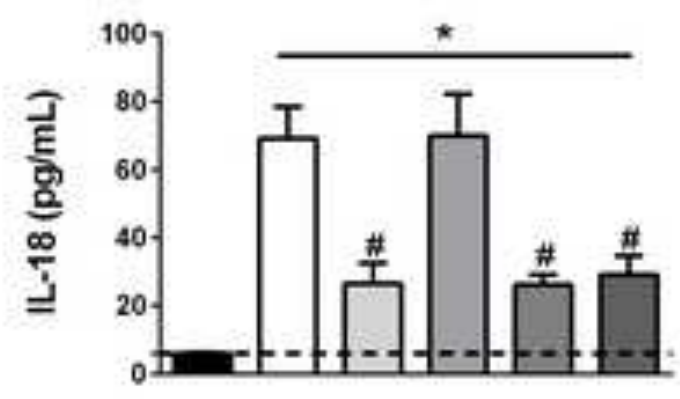

$\mathbf{F}$
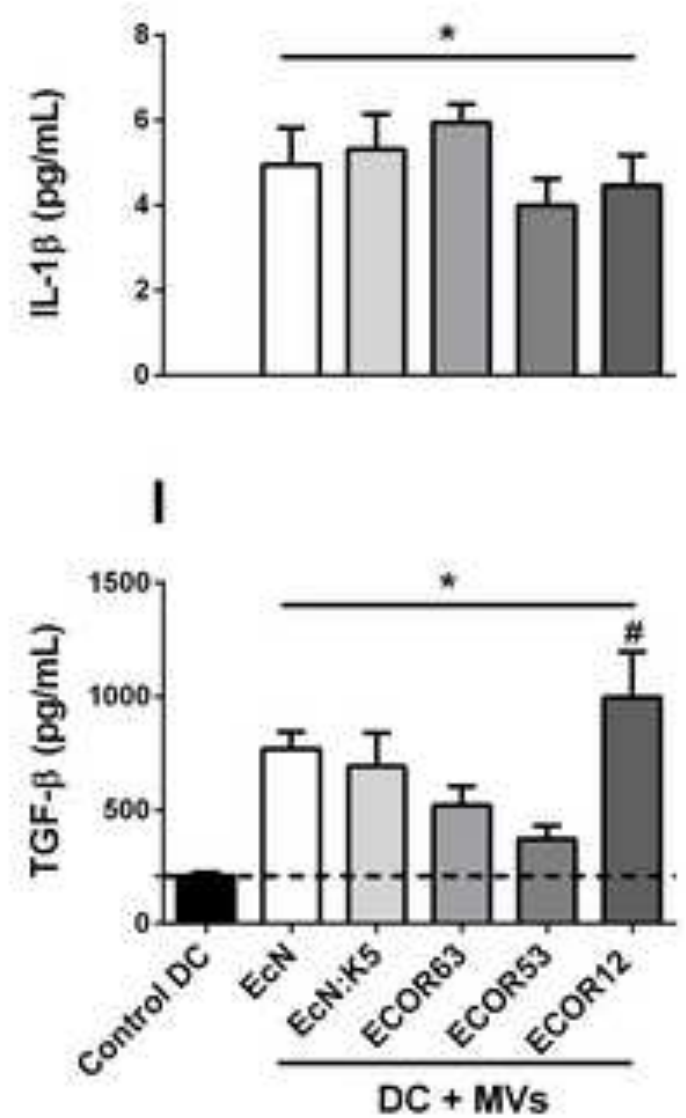
Figure 4

Click here to download high resolution image

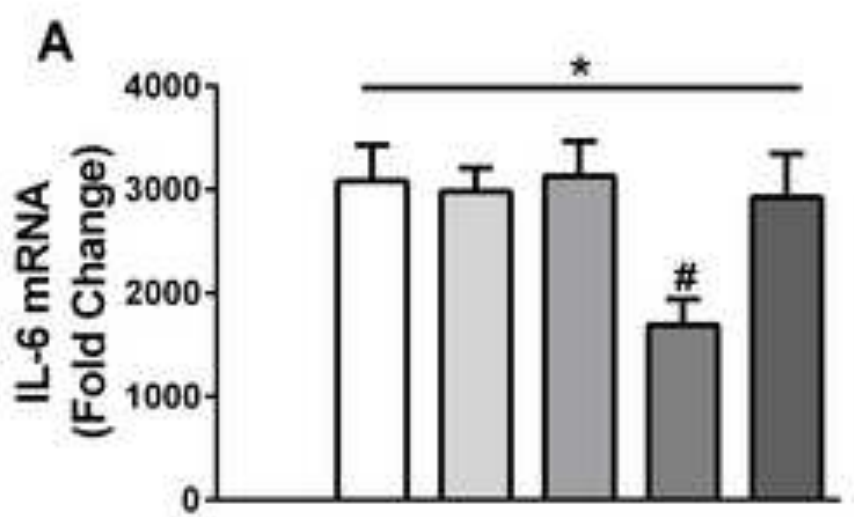

B

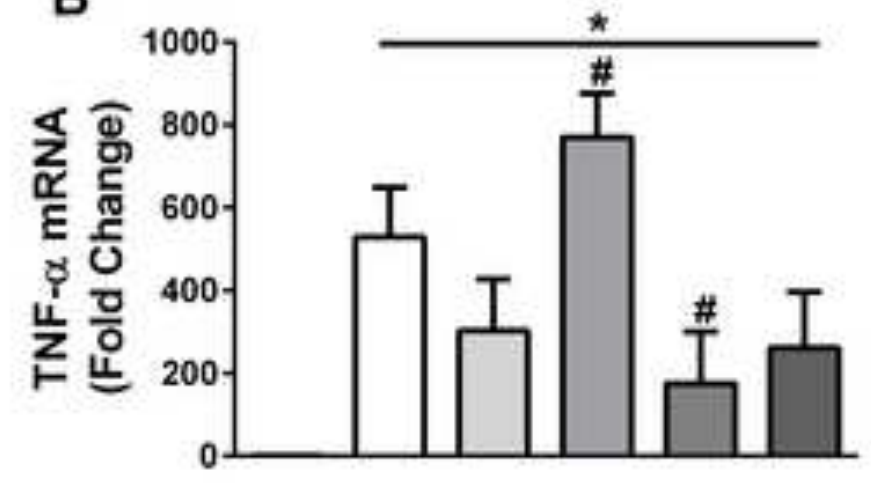

C
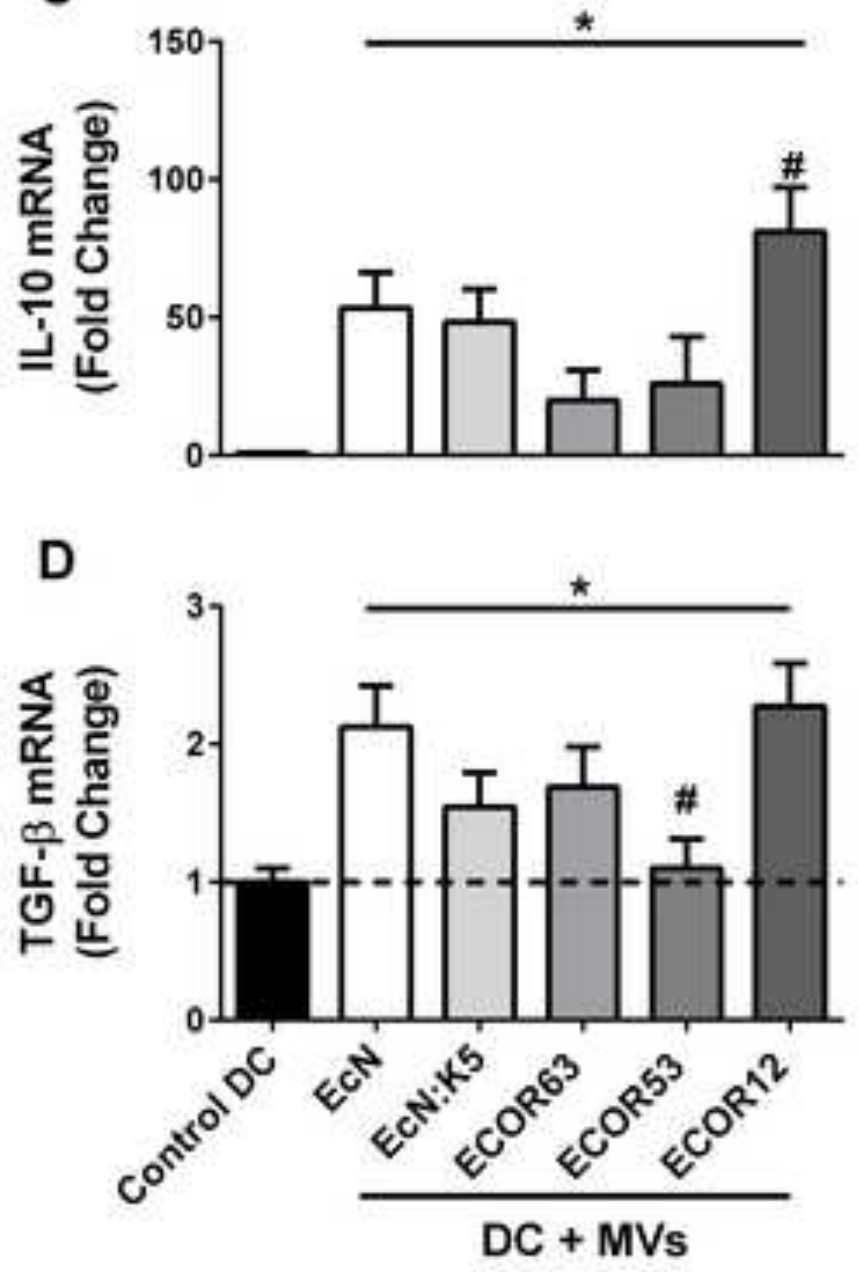

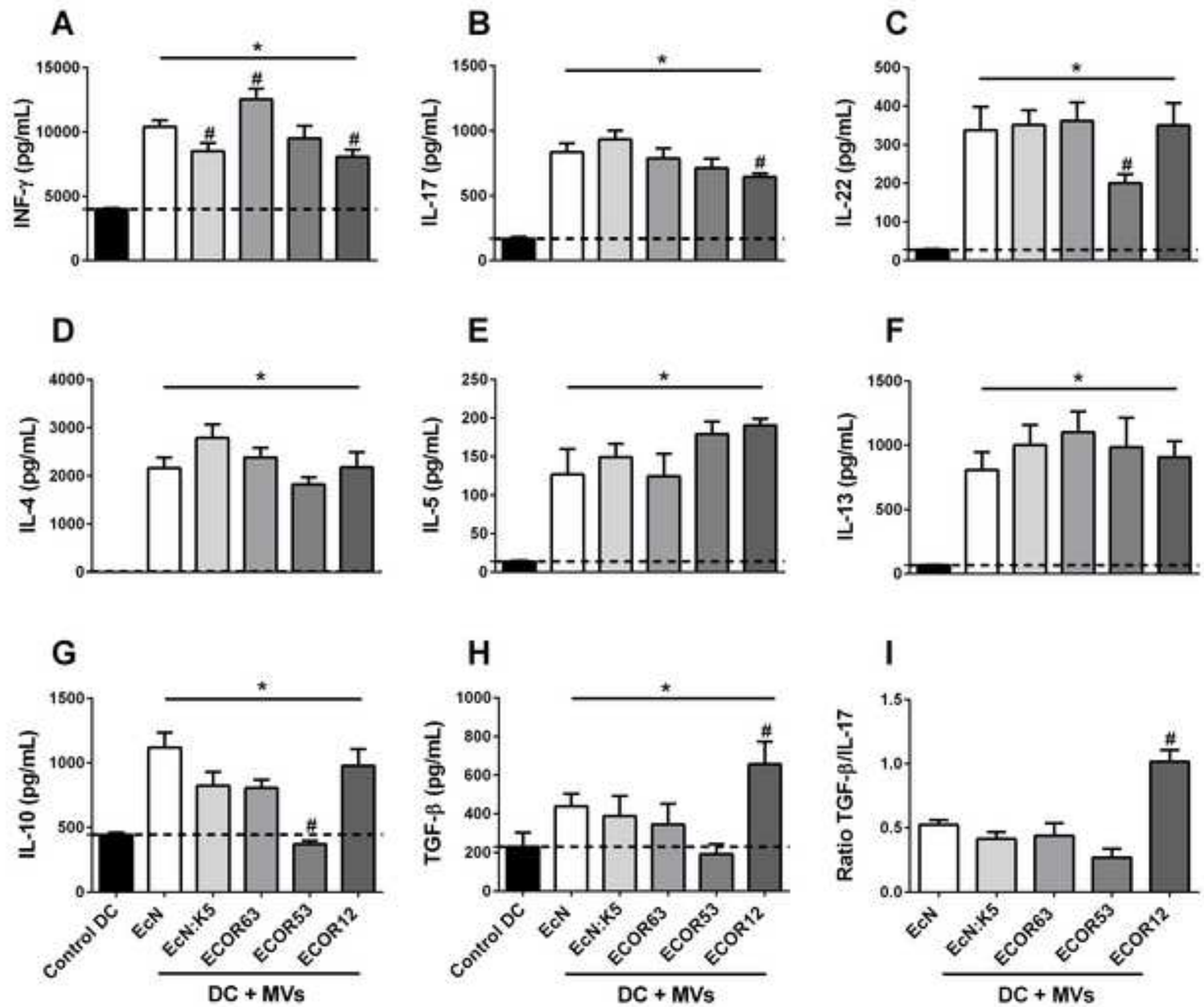


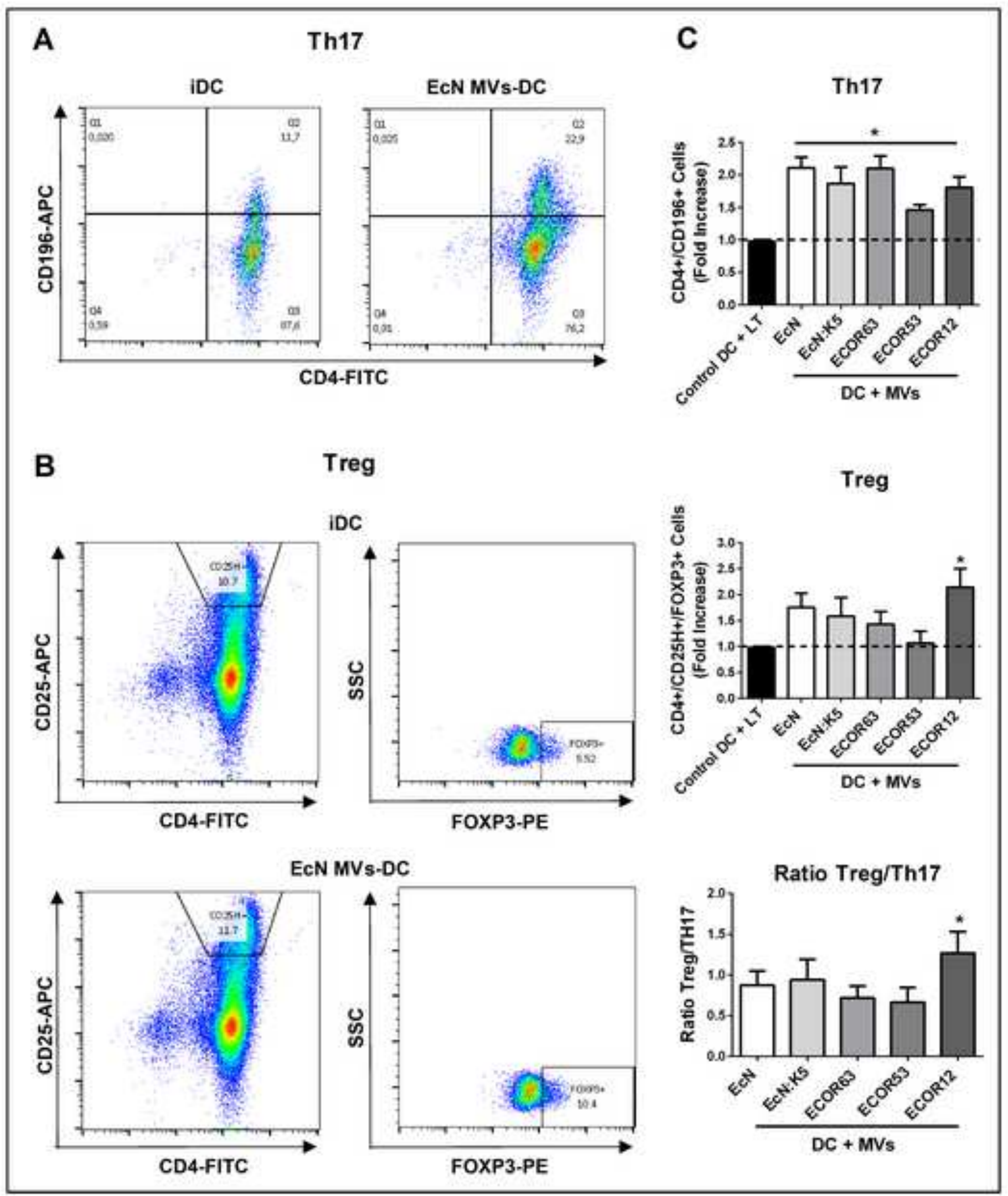


Figure 7
Click here to download high resolution image
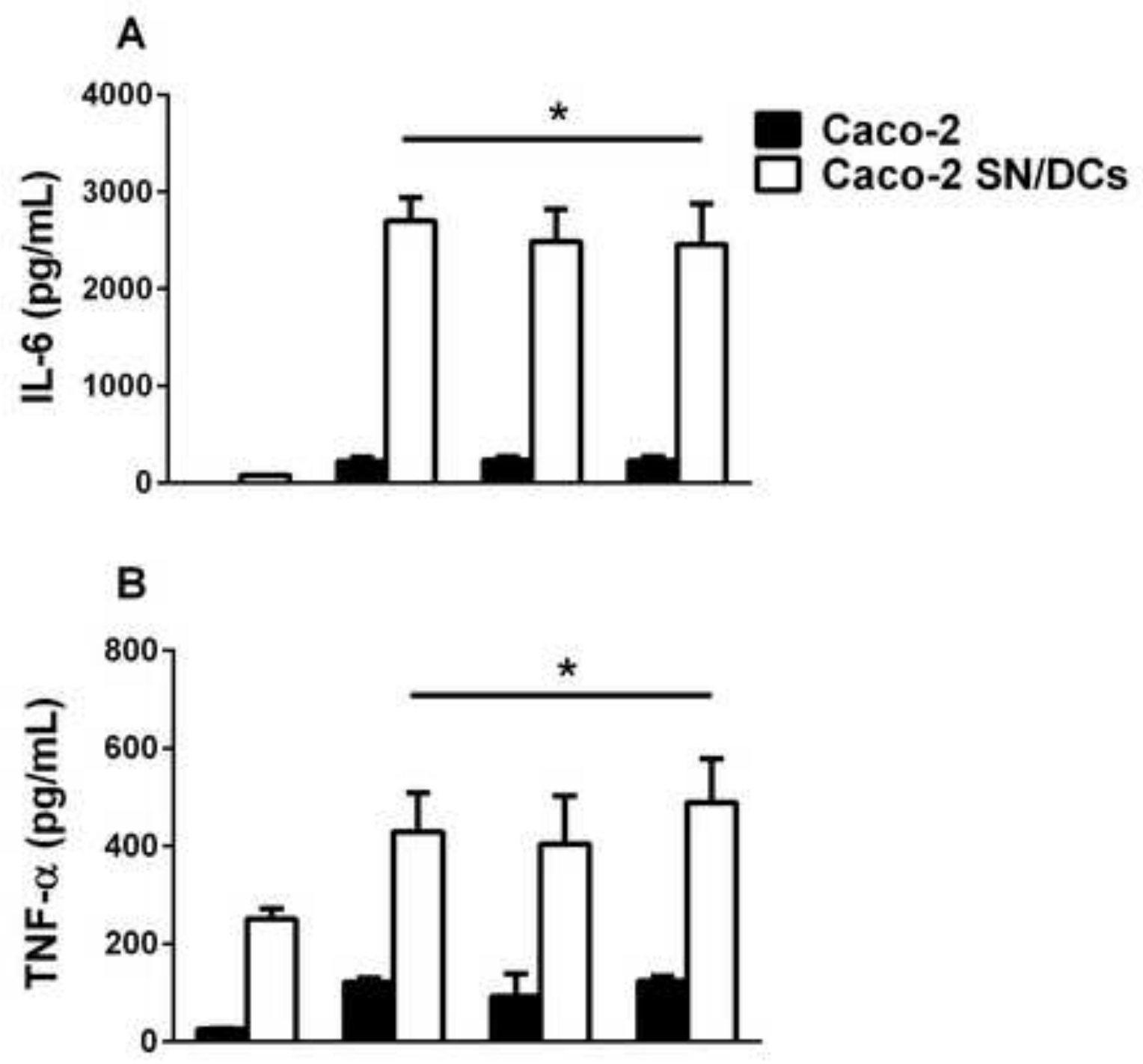

C

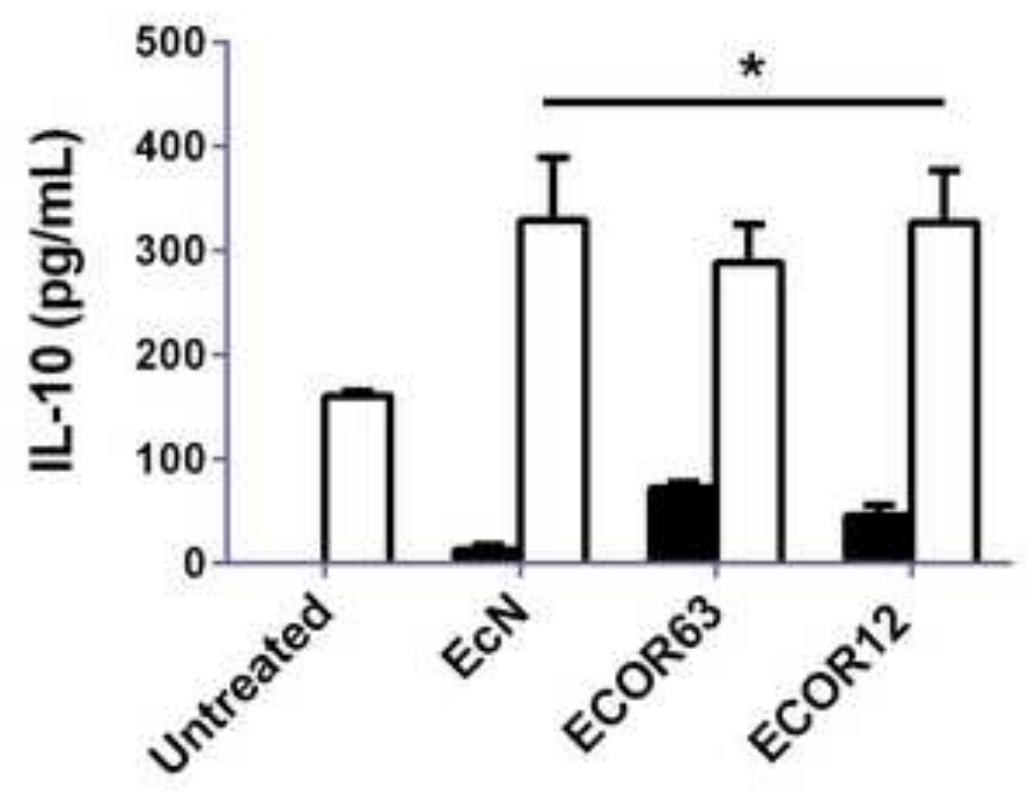



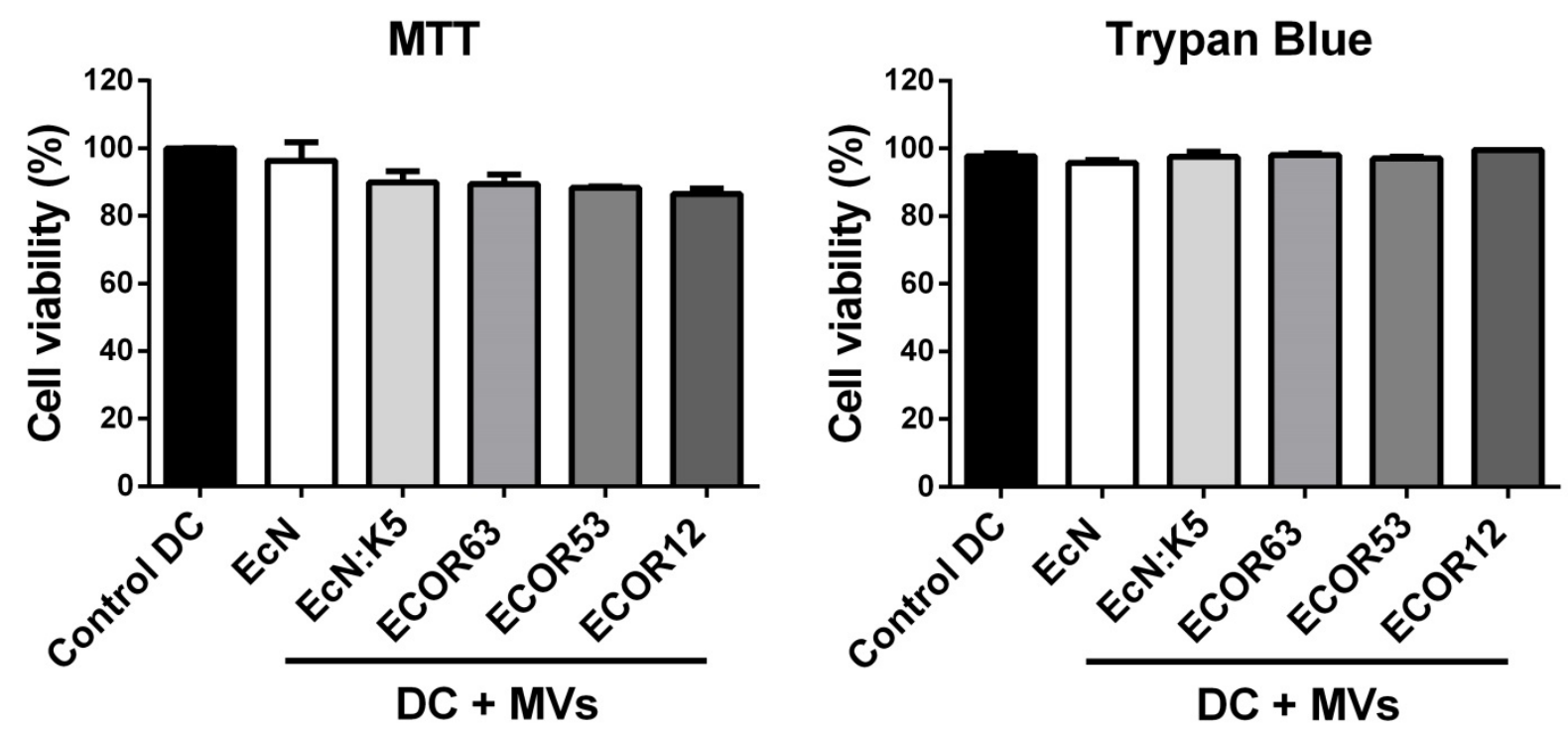

Figure S1. Cell viability of mo-DCs stimulated for $24 \mathrm{~h}$ with MVs $(10 \mu \mathrm{g} / \mathrm{ml})$ of the indicated strains assessed by the MTT and trypan blue assays Values are means \pm standard error from three independent experiments. 


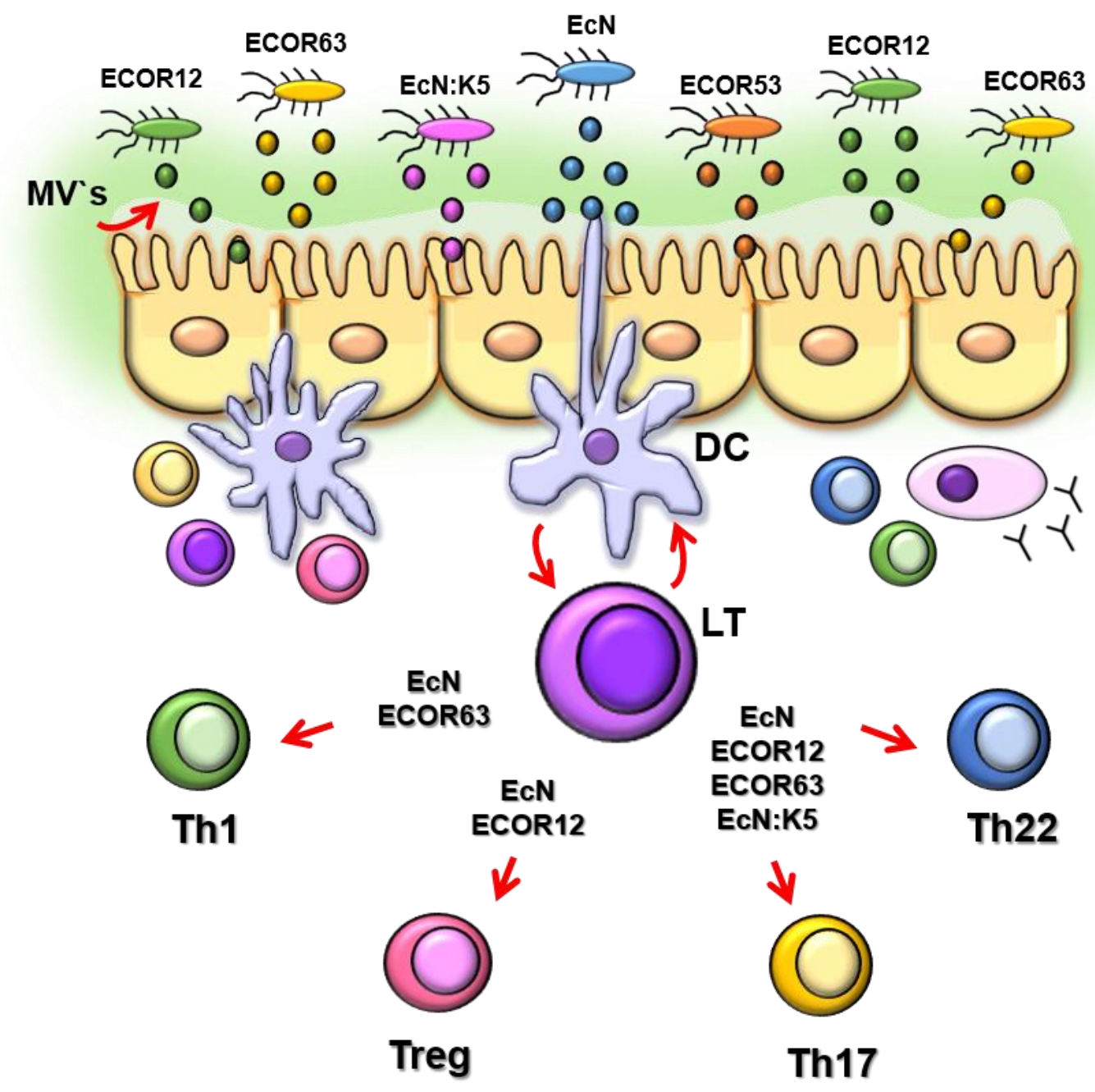




\section{Conflict of Interest Statement}

The authors declare that the research was conducted in the absence of any commercial or financial relationships that could be construed as a potential conflict of interest. 


\section{Ethics statement}

Human peripheral blood mononuclear cells were isolated from fresh buffy coats of healthy donors, provided by the "Banc de Sang i Teixits" of Barcelona according to the signed agreement with the Institution. The use of anonymous, non-identifiable human samples was approved by the Bioethics Committee of the University of Barcelona (Institutional Review Board:1R800003099). 